\title{
Relaxation volumes of microscopic and mesoscopic irradiation-induced defects in tungsten
}

\section{Mason, Daniel R.}

2019-08-21

Mason, D R, Duc Nguyen-Manh, Marinica , M-C , Alexander , R , Sand , A E \& Dudarev , $S \mathrm{~L} 2019$, ' Relaxation volumes of microscopic and mesoscopic irradiation-induced defects in tungsten ' , Journal of Applied Physics , vol. 126 , no. 7 , 075112 . https://doi.org/10.1063/1.5094852

http://hdl.handle.net/10138/318087

https://doi.org/10.1063/1.5094852

acceptedVersion

Downloaded from Helda, University of Helsinki institutional repository.

This is an electronic reprint of the original article.

This reprint may differ from the original in pagination and typographic detail.

Please cite the original version. 


\section{Relaxation volumes of microscopic and mesoscopic irradiation-induced defects in tungsten}

Article in Journal of Applied Physics · August 2019

DOI: $10.1063 / 1.5094852$

CITATIONS

0

6 authors, including:

Daniel R Mason

Culham Centre for Fusion Energy

46 PUBlications 607 CITATIONS

SEE PROFILE

R Rebecca Alexander

1 Université de Lille

6 PUBLICATIONS 18 CITATIONS

SEE PROFILE

Some of the authors of this publication are also working on these related projects:

Kinetic Monte Carlo Algorithms for Dynamic Processes in Metals View project

Project Automated TEM analysis View project
READS

116

Mihai-Cosmin Marinica

Atomic Energy and Alternative Energies Commission

44 PUBLICATIONS 1,003 CITATIONS

SEE PROFILE

Andrea E Sand

University of Helsinki

42 PUBLICATIONS 415 CITATIONS

SEE PROFILE 


\title{
Relaxation volumes of microscopic and mesoscopic irradiation-induced defects in tungsten
}

\author{
Daniel R. Mason, ${ }^{1}$ Duc Nguyen-Manh,,${ }^{1,2}$ Mihai-Cosmin Marinica, ${ }^{3}$ Rebecca Alexander, ${ }^{4}$ Andrea E. Sand ${ }^{5}$ and \\ Sergei L. Dudarev ${ }^{1,2}$ \\ 1) CCFE, UK Atomic Energy Authority, Culham Science Centre, Oxfordshire OX14 3DB, \\ $U K$ \\ ${ }^{2)}$ Department of Materials, University of Oxford, Parks Road, Oxford OX1 3PH, \\ $U K$ \\ ${ }^{3)}$ DEN-Service de Recherches de Metallurgie Physique, CEA, Universite Paris-Saclay, F-91191, Gif-sur-Yvette, \\ France \\ ${ }^{4)}$ Metallurgie Physique et Genie des Materiaux, UMET, CNRS, UMR 8207, Universite de Lille, F-59000, Lille, \\ France \\ 5) Department of Physics, University of Helsinki, P.O. Box 43, FI-00014, Helsinki, \\ Finland
}

(Dated: 24 July 2019)

The low energy structures of irradiation-induced defects in materials have been extensively studied over several decades, as these determine the available modes by which a defect can diffuse or relax, and how the microstructure of an irradiated material evolves as a function of temperature and time. Consequently many studies concern the relative energies of possible defect structures, and empirical potentials are commonly fitted to, or evaluated with respect to these. But recently [Dudarev et al. Nuclear Fusion 2018], we have shown that other parameters of defects not directly related to defect energies, namely their elastic dipole tensors and relaxation volumes, determine the stresses, strains and swelling of reactor components under irradiation. These elastic properties of defects have received comparatively little attention. In this study we compute relaxation volumes of irradiation-induced defects in tungsten using empirical potentials, and compare to density functional theory results. Different empirical potentials give different results, but some clear potential-independent trends can be identified. We show that the relaxation volume of a small defect cluster can be predicted to within $10 \%$ from its point-defect count. For larger defect clusters we provide empirical fits as a function of defect cluster size. We demonstrate that the relaxation volume associated with a single primary-damage cascade can be estimated from the primary knock-on atom energy. We conclude that while annihilation of defects invariably reduces the total relaxation volume of the cascade debris, there is still no conclusive verdict about whether coalescence of defects reduces or increases the total relaxation volume.

Keywords: Radiation Induced Defects, Relaxation Volumes

\section{INTRODUCTION}

Just as it has been long acknowledged that the effect of radiation on materials is inherently multi-scale both in time- and spatial- dimension, so it is accepted that to model these effects requires transfer of high quality data from one model to the next ${ }^{1}$. The form of the data required by a coarse-grained model varies, and a typical workflow in nuclear materials modelling involves finding structural information about individual defects from Density Functional Theory $(\mathrm{DFT})^{2,3}$, information about the cascade generation process from Molecular Dynamics $(\mathrm{MD})^{4-6}$, and about cascade evolution from object or atomistic Kinetic Monte Carlo (KMC) ${ }^{7-9}$ or Cluster Dynamics $(\mathrm{CD})^{10,11}$ simulations. This has proved successful for modelling the experimentally observed size and distribution of irradiation-induced defects formed in pure single crystalline materials ${ }^{9,12}$.

Recently we have shown that it is possible to compute stresses and strains in reactor components on the macroscopic scale of centimetres and metres from the distribution of irradiation-induced defects ${ }^{13}$. As a source term, this model only requires the spatially varying density of relaxation volumes of defects, and so allows the direct simulation of volumetric radiation-induced swelling and the associated stresses from an atomistic or object-based model. At the nanoscale, lattice swelling resulting from the accumulation of radiation defects is experimentally measurable using Micro-Laue diffraction ${ }^{14,15}$. Furthermore, X-ray diffraction measurements show that negative lattice strain develops due to the accumulation of vacancies in a material ${ }^{16}$. On a macroscopic scale, predicting the stress state of reactor components arising from irradiation is fundamental to the successful engineering design of a nuclear fission or fusion power plant ${ }^{17-19}$. One outstanding issue, which this paper is intended to address, is to have good quality data for the relaxation volumes of a variety of lattice defects, as while accessible from simulation for years, reporting this information has been somewhat neglected in favour of establishing accurate values of formation energies and low-energy-state structures. For example, a comprehensive compilation of data on relaxation volumes and relaxation volume tensors of individual self-interstitial and vacancy point de- 
fects derived from DFT calculations performed for all the bcc metals in the Periodic Table has been reported only recently ${ }^{13,20-22}$. Data for the relaxation volumes of point defects available prior to these studies were derived mostly from experimental measurements and semiempirical potential calculations ${ }^{23-27}$ and exhibited a degree of variability associated with experimental uncertainties or the choice of interatomic interaction potentials. Accurate $a b$ initio studies of relaxation volumes of defects mostly involved vacancies in simple or noble metals $^{13,25,28-33}$.

Here we focus our attention on a single material, tungsten. Tungsten has been chosen as a divertor material for ITER ${ }^{18,34,35}$ as it has a high melting point, high thermal conductivity and high resistance to sputtering. For our purposes tungsten is also well-suited to this preliminary study of relaxation volumes as it is nearly elastically isotropic ${ }^{36}$ at low temperatures. This simplifies the expressions needed for an elastic analysis, but is by no means a requirement of the atomistic techniques used here ${ }^{20}$, and the data we present has no assumption of isotropy. The finite temperature calculation of the Helmholtz free energy and an anisotropic elasticity analysis are beyond the scope of the current paper. In section II A, we compute the relaxation volumes of small defect clusters, and compare the results obtained with several embedded atom (EAM) potentials with those derived from DFT. In section II B, we move on to larger lattice defect objects. As the number of configurational degrees of freedom becomes very large, we focus on a standard set of idealised dislocation loops and voids, which often form a basis set for object kinetic Monte Carlo (okMC) or Cluster Dynamics (CD) simulations. In section II C we introduce the orientation-dependent anisotropy of the relaxation. In section IID we consider interacting groups of defect clusters generated in high energy collisions simulated by MD. Our goal is to understand the complexlooking stress fields which arise in irradiated metals (see figure 1) in terms of the simpler stress fields of their constituent parts, which can then be used to predict volumetric swelling from the output of existing microstructural evolution codes such as okMC or CD.

Early estimates of formation volumes for point defects in tungsten were established by Johnson ${ }^{38}$, using an empirical potential. This work found a negative relaxation volume for a vacancy $\left(-0.21 \Omega_{0}\right.$, where $\Omega_{0}$ is the atomic volume), and a positive relaxation volume for the interstitial $\left(+1.13 \Omega_{0}\right)$. DFT calculations by Kato et al. ${ }^{39}$ confirmed the relaxation volume for the vacancy as negative, at $-0.34 \Omega_{0}$, a figure which has since been reproduced several times by independent DFT calculations. The relaxation volume of a $1 / 2\langle 111\rangle$ interstitial defect was shown to be large and positive in DFT calculations at $+1.68 \Omega_{0}{ }^{15}$ in a small $4 \times 4 \times 4$ supercell, later confirmed by other DFT calculations $^{20,21}$.

We demonstrate that empirical potentials give varying results for the relaxation volumes of irradiation defects. This is an expected result, as these properties of defects

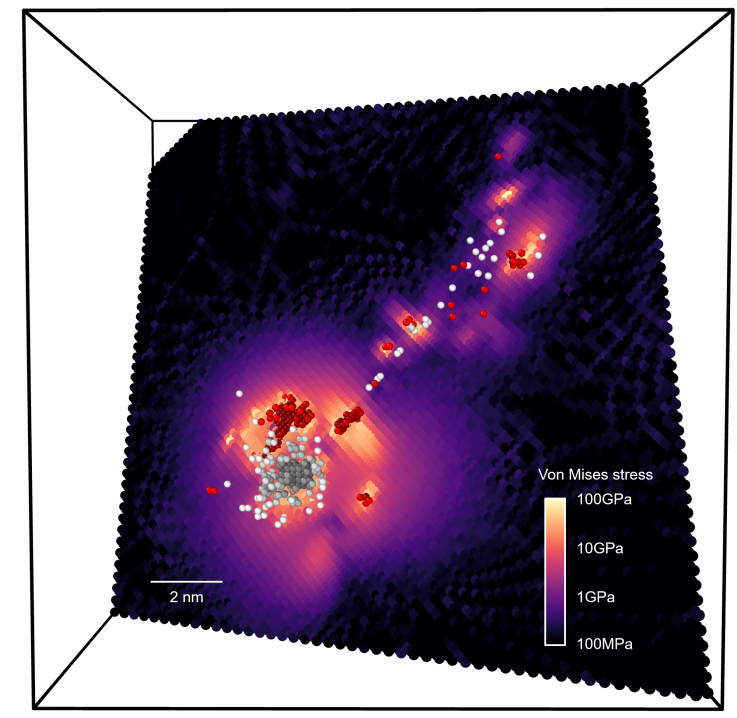

FIG. 1. A typical configuration of defects produced by a collision cascade event in tungsten, initiated by a $150 \mathrm{keV}$ PKA, and simulated using the method described in section IID. Vacancies (white spheres), and interstitials (red), were identified using a Wigner-Seitz defect analysis ${ }^{37}$. The von Mises stress in a [211] plane intersecting the cascade is also shown. Note that close to the defects the stresses can be as high as $100 \mathrm{GPa}$, comparable to the shear modulus $(\mu=160 \mathrm{GPa})$. In the study below, we analyse the complex stress fields of individual defects and clusters of defects formed in cascades, similar to those shown in this figure.

were never originally used as input data during the parameterization of potentials. The relaxation volumes do, however, show systematic trends across potentials. It is beyond the scope of this paper to provide a comprehensive comparison of empirical potentials, instead our comparison will focus on three empirical potentials, which should give an indication of the possible variation.

- The Derlet-Nguyen-Manh-Dudarev (DND) potential $^{40}$, has been shown to produce cascade structures that are a good match to experiment $^{6,12,41-43}$.

- One of the four potential parameterizations developed by Marinica et al. (CEA-4) $)^{44}$, which has a good balance between the predicted point- and extended defect properties. This potential was developed from the DND and AM04 ${ }^{45}$ potentials with additional fitting to the forces on atoms in disordered systems.

- A new potential parameterization by some of the authors $(\mathrm{MNB})^{46}$, which is a development of the smooth and highly-transferable Ackland-Thetford potential $^{47,48}$, corrected to give better properties for vacancy-type structures.

We present simple empirical formulae for the relaxation volumes of defects that might be used for predicting 
stresses and strains in engineering components containing these defects ${ }^{13}$. As tungsten is (nearly) elastically isotropic, it suffices to present results in terms of a single relaxation volume, and the relaxation volume anisotropy parameter, defined as the ratio of the smallest to largest partial relaxation volumes. Simple formulae are required to switch between this representation and the full dipole tensor for the defect.

Finally in section III we compare our results with analytical formulae derived using linear elasticity and surface energies, consider the differences between the empirical potentials used and discuss routes for making predictions for complex microstructures, and in section IV we compare the predictive power of our results to earlier studies.

\section{RELAXATION VOLUME OF DEFECT STRUCTURES}

Relaxation volumes can be computed for isolated defects and relaxed cascade configurations using several methods. As a validating convergence study, we compare three methods: the stress method, the cell relaxation method, and the free surfaces method:

- The stress method. The atom positions are relaxed in a periodic supercell, with the vectors defining the supercell repeat fixed. The stress is computed on each atom and summed to give a single tensor for the cell. The relaxation of a body free from surface tractions due to the defect is predicted from this stress using linear elasticity theory. The lattice vectors of the simulation cell never need to be updated.

- The cell relaxation method. As with the stress method, the atoms are relaxed in a periodic supercell, and the stress is computed. From this a strain is computed, but in contrast to the stress method this is then applied to the supercell, changing its shape and volume. The vectors defining the supercell repeat are updated and the relaxation process is repeated until convergence, where the macroscopic stress vanishes. This iterative process of relaxing first the atoms and then the cell differentiates this method from the stress method. We note here that there is no a priori reason why the minimum energy structure of a defect with one set of lattice vectors is the same as the minimum with a different set.

- The free surface method. A large sphere of atoms is constructed and relaxed, producing a body with explicitly free surfaces ${ }^{49}$. Then a single defect is constructed inside the sphere and the entire structure is relaxed again. The volume of a (distorted) spheroid after relaxation is more difficult to compute than with a periodic supercell, as it is not clear where the surface should be drawn. However, the volume enclosed by the convex hull of the atoms $V_{\text {hull }}$ is easy to compute using qhull ${ }^{50}$. From this we can estimate that the volume of the sphere is $V_{\text {spheroid }}=V_{\text {hull }}(R+r)^{3} / R^{3}$, where $R$ is the maximum radius of atom positions on the convex hull, and $r=\mathrm{a}_{0} / 4$ is one quarter the lattice parameter, which is half the distance between $\{100\}$ planes in a bcc crystal. $^{51}$ We include this non-standard ( and sub-optimal ) brute-force method as a validation that the linear elasticity approach followed in this work truly predicts the relaxation volume in a body with explicitly free surfaces.

In this work we do not consider the method of Kanzaki forces $^{52,53}$, or the method of matching displacements ${ }^{54,55}$ for estimating relaxation volumes using the harmonic region of the crystal only.

To compute the stress due to a defect, and hence the strain in an elastic medium we compute the dipole tensor as the integrated stress over the cell ${ }^{21,56,57}$

$$
P_{i j}=-\int_{V} \sigma_{i j}(\mathbf{r}) \mathrm{d}^{3} \mathbf{r} .
$$

In an atomistic simulation where the energy is more easily accessible than the stress, we can compute this quantity as the derivative of total energy with respect to a homogeneous body strain,

$$
P_{i j} \equiv-\frac{\partial E}{\partial \epsilon_{i j}^{0}} .
$$

The dipole tensor may also be expressed in terms of a symmetric dual tensor, $\Omega_{k l}$, characterizing the volumetric relaxation of the defect, and defined by the equation ${ }^{13,20}$

$$
P_{i j} \equiv C_{i j k l} \Omega_{k l},
$$

where $C_{i j k l}$ are the elements of the fourth-rank tensor of elastic constants. From this dual tensor we can find the relaxation volume $\Omega_{r e l}$ of the defect characterizing the volumetric relaxation of an elastic body free from surface tractions ${ }^{56}$,

$$
\Omega_{r e l}=\operatorname{Tr} \Omega \equiv \sum_{i=1}^{3} \Omega^{(i)}
$$

where $\Omega^{(i)}$ are the three eigenvalues of the tensor $\Omega_{k l}$, corresponding to the three partial relaxation volumes. Hence we can find the elastic relaxation volume of the defect using a constant-volume calculation, if the elastic constants and dipole tensor of the defect are computed.

For an empirical potential, $P_{i j}$ can be computed simply and analytically. For the embedded-atom form we compute the energy as a sum over pairwise and manybody contributions: $E=\sum_{a} V_{a}+F\left[\rho_{a}\right]$, where $V_{a}=$ $1 / 2 \sum_{b} V\left(r_{a b}\right)$ is a pairwise interaction, $\rho_{a}=\sum_{b} \phi\left(r_{a b}\right)$ models the embedding electron density and $F[\rho]$ is the many-body embedding energy. The dipole tensor is

$$
P_{i j}=-\sum_{a, b}\left(\left.\frac{1}{2} \frac{\partial V}{\partial r}\right|_{r_{a b}}+\left.\left.\frac{\partial F}{\partial \rho}\right|_{\rho_{a}} \frac{\partial \phi}{\partial r}\right|_{r_{a b}}\right) \frac{r_{a b, i} r_{a b, j}}{r_{a b}},
$$


where $r_{a b}$ is the separation between atoms $a$ and $b$ and $r_{a b, i}$ is its $i^{\text {th }}$ Cartesian coordinate. This is a simple sum over atoms and their neighbours, using the same first derivatives as a force calculation, and so is trivially implemented in any MD or atomistic MS code. The (fourthrank) elastic constant tensor can be computed analytically from the second derivative of energy with respect to strain ${ }^{47}$. The computed elastic constants are given in table II.

A plot showing the convergence of the numerical procedure for computing the relaxation volume of a 19interstitial loop with system size is shown in figure 2 . Extrapolating to infinite system size suggests all three methods converge to the same result, though at any given finite system size there will be an error, typically scaling as inverse system size $1 / n$ ( voids relaxed with explicitly free surfaces being an exception, converging as the inverse radius of the free sphere $1 / R$. ) This $1 / n$ convergence was also observed recently by Varvenne \& Clouet $^{55}$, who attributed this leading error term to the interaction between periodic images. Some indicative data are also given in table I proving that the stress method with large supercells is suitable for the structures considered in this work. Relaxation volumes computed with the EAM potentials in this paper are computed using the dipole tensor method at a converged supercell size. Relaxation volumes computed with DFT were computed using the full cell relaxation method.

\section{A. Small defect structures}

In this section we compute the elastic properties of small defect clusters. For sufficiently small clusters we can perform a fairly comprehensive survey of possible structures, and so find the bounds of the variation of the relaxation volumes. The question of which are the most significant set of small clusters to use is rather more difficult. Generally, for a cluster containing $N$ point defects, we might expect to find only a small number of structures within a few $\mathrm{meV}$ of the ground state. At low temperatures these are the only ones which need to be considered in equilibrium. But radiation damage is an inherently non-equilibrium process. The system can generally reduce its internal energy by coalescing clusters, and the true equilibrium is only found when nearly all defects have recombined or diffused to sinks. The structures that may actually be found at some time after the cooling of a displacement cascade could therefore be, briefly at least, rather exotic.

Randomly generated interstitial clusters were generated by placing $N$ additional atoms into an otherwise perfect crystal, then relaxing. The extra atoms were placed at random into $[l, m, n] \mathrm{a}_{0} / 4$ crystal positions, with $0 \leq \operatorname{lm} n<4$, with the constraint that an atom was not placed if another was already within a distance $\mathrm{a}_{0} / 2$. All extra atoms were placed in a central block of $2 \times 2 \times 2$ unit cells. The relaxation volumes as a function of formation
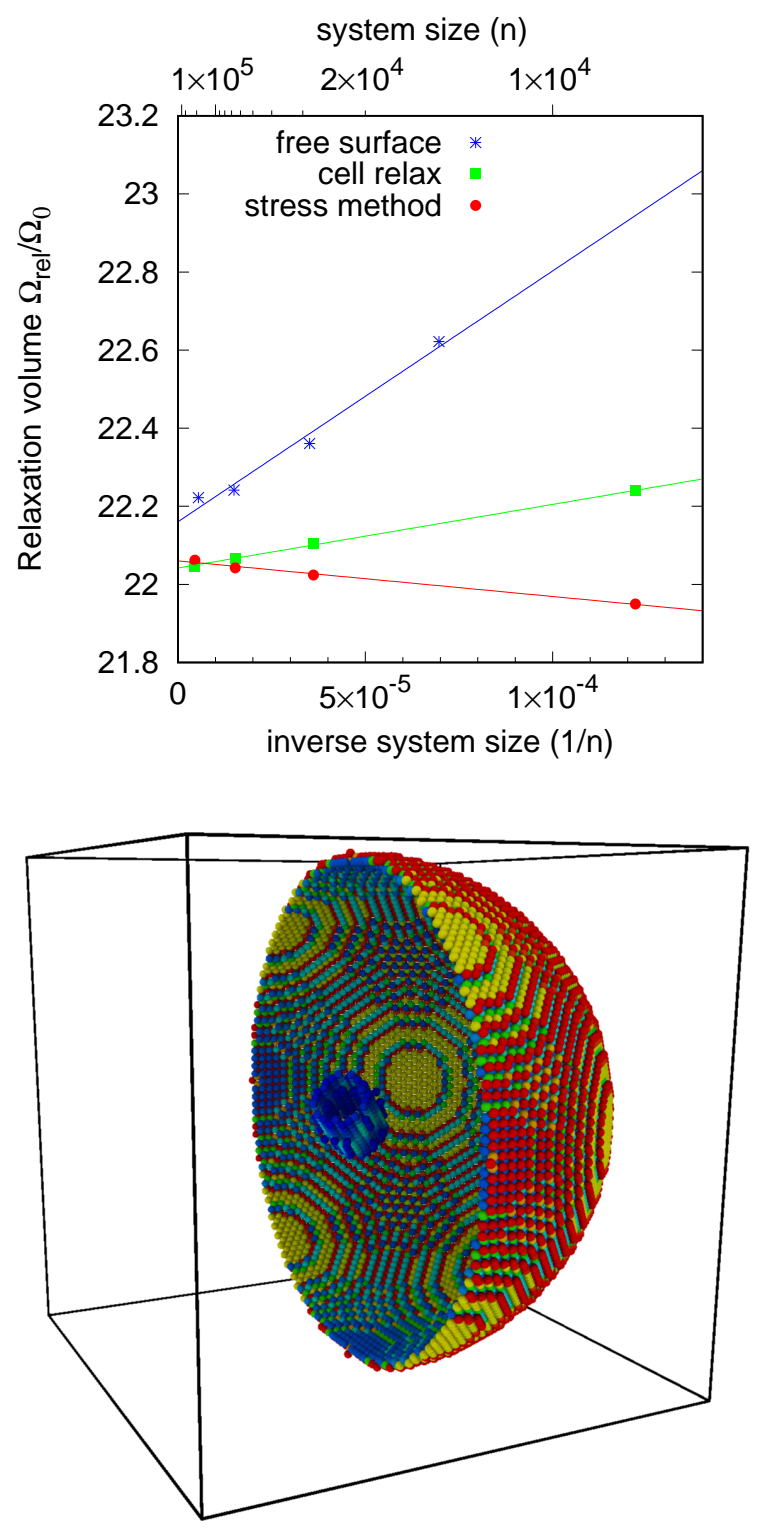

FIG. 2. Above: Example convergence study for relaxation volume of the 19 interstitial cluster computed using three methods. All three methods converge to very similar values, with a system size error inversely proportional to the number of atoms in the simulation cell $n$. Best fit lines are indicated to guide the eye. Calculations were performed with the MNB potential. Other potentials and other defects show qualitatively similar results. Below: Geometry used for free-surface calculations. An unsupported free sphere of atoms was constructed and relaxed, then a single defect was generated in the centre and the atoms relaxed again. The volume before and after the defect was placed was computed using qhull ${ }^{50}$. One half of the sphere is shown ${ }^{37}$, together with high energy atoms of the defect. Atoms are coloured by excess potential energy from $0 \mathrm{eV}$ (blue) to $2 \mathrm{eV}$ (red). Atoms with energy under $0.1 \mathrm{eV}$ are not shown. In this image a 55 interstitial loop is embedded in a sphere of 180000 atoms, and relaxed with the MNB potential.

energy and cluster size are presented in figure 3 . We have 


\begin{tabular}{l|ccc} 
& cell relaxation & stress method & free surfaces \\
\hline $1 \mathrm{v}$ & -0.367 & -0.368 & -0.350 \\
$15 \mathrm{v}_{s p h}$ & -3.599 & -3.625 & -3.731 \\
$65 \mathrm{v}_{s p h}$ & -9.658 & -9.760 & -10.351 \\
$259 \mathrm{v}_{s p h}$ & -21.634 & -21.806 & -24.291 \\
\hline $1 \mathrm{i}_{\langle 111\rangle}$ & 1.399 & 1.400 & 1.415 \\
$19 \mathrm{i}_{\langle 111\rangle}$ & 22.045 & 22.062 & 22.222 \\
$55 \mathrm{i}_{\langle 111\rangle}$ & 60.325 & 60.250 & 60.705 \\
$199 \mathrm{i}_{\langle 111\rangle}$ & 209.946 & 209.283 & 210.947
\end{tabular}

TABLE I. Relaxation volumes for vacancy defects ( monovacancy, and 15,65,259 vacancy spheres ) and interstitial defects ( $1 / 2\langle 111\rangle$ dumbbell, 19,55,199 $\langle 111\rangle$ interstitial loops ). The volumes are expressed in units of atomic volume, computed using the MNB potential. The cell relaxation and stress method calculations used a $48 \times 48 \times 48$ unit cell supercell. The free surfaces method used a sphere with an initial radius of 28 unit cells. It is clear that the stress method does indeed reproduce the relaxation volume of the defect in a body with traction free surfaces at large system size, and so is an acceptable faster alternative to the full cell relaxation calculation.

\begin{tabular}{|c|c|c|c|c|c|c|}
\hline method & $a_{0}$ & $c_{11}$ & $c_{12}$ & $\gamma_{\langle 110\rangle} \gamma_{\langle 100\rangle}$ & $\gamma_{\langle 211\rangle} \gamma_{\langle 111\rangle}$ & $\gamma$ \\
\hline DFT & 3.186 & 3.229 & 1.2240 .888 & $0.200 \quad 0.245$ & $0.215 \quad 0.219$ & 0.229 \\
\hline MNB & 3.1652 & 3.222 & 1.2630 .998 & $0.218 \quad 0.239$ & $0.241 \quad 0.257$ & \begin{tabular}{l|l}
7 & 0.234
\end{tabular} \\
\hline DND & 3.1652 & 3.3881 & 1.3041 .031 & $\begin{array}{lll}0.150 & 0.187\end{array}$ & $0.185 \quad 0.161$ & \begin{tabular}{l|l}
1 & 0.174
\end{tabular} \\
\hline CEA4 & 3.14339 & 3.265 & 1.2621 .004 & $\begin{array}{lll}0.157 & 0.183\end{array}$ & $\begin{array}{lll}3 & 0.187 & 0.201\end{array}$ & 0.177 \\
\hline expt & 3.1652 & 3.324 & 1.2791 .018 & & & 0.229 \\
\hline methoo & $s_{\langle 1}$ & 10) & $s_{\langle 100\rangle}$ & $s_{\langle 211\rangle}$ & $s_{\langle 111\rangle}$ & $s$ \\
\hline$\overline{\mathrm{DFT}}$ & 0.375 & 0.253 & $\begin{array}{lll}0.296 & 0.286\end{array}$ & 0.4650 .434 & 0.3130 .313 & $\overline{0.360}$ \\
\hline MNB & 0.330 & 0.215 & $\begin{array}{lll}0.229 & 0.229\end{array}$ & 0.2630 .252 & $\begin{array}{lll}0.215 & 0.214\end{array}$ & 0.253 \\
\hline DND & 0.150 & -0.008 & $\begin{array}{lll}0.291 & 0.275\end{array}$ & $0.144 \quad 0.044$ & 0.1790 .022 & 0.129 \\
\hline CEA4 & 0.125 & 0.018 & $\begin{array}{lll}0.210 & 0.210\end{array} \mid$ & $0.075 \quad 0.056$ & $\begin{array}{lll}0.064 & 0.063\end{array}$ & 0.100 \\
\hline
\end{tabular}

TABLE II. The lattice constant $\mathrm{a}_{0}$ (in $\AA$ ) and elastic constants (in $\mathrm{eV} / \AA^{3}$ ) of bcc tungsten, from ${ }^{15}$. The surface energies $\gamma_{h k l}$ of bcc tungsten for the $[h k l]$ surface are given in $\mathrm{eV} / \AA^{2}$. The principal surface stresses $s_{h k l}$ for the $[h k l]$ surface ( two non-zero eigenvalues of $\left.s_{i j}=\gamma \delta_{i j}+\partial \gamma / \partial \epsilon_{i j}\right)$ of bcc tungsten are given in $\mathrm{eV} / \AA^{2}$. Average surface energy $\gamma$ computed using equation A4, and average surface stress $s$ using the mean of the principal eigenvalues in equation A4. Experimental elastic constants from $\operatorname{ref}^{58}$, and surface energy from $\operatorname{ref}^{59}$.

not put results from CEA4 on this scatter plot. CEA4 has a good deal of structure in its potential, which allows for a very large number of metastable high energy defect clusters to form. On figure 3, this would appear as an almost structureless cloud and we conclude our method of randomly generating interstitial clusters is not suited to this potential.

DFT calculations of small interstitial clusters were performed for this work using the VASP ab initio simulation code, using the PAW method ${ }^{60-62}$ with semi-core electrons included through the use of pseudo-potentials. It is important to emphasize that the inclusion of semi-core electrons in the valence states has a significant effect on the predicted formation energies of self-interstitial atom (SIA) defects for all the bcc transition metals ${ }^{2,63,64}$, and play important role on the quality of inter-atomic potential in predicting non-equilibrium properties in tungsten from cascade simulations ${ }^{65}$. Exchange-correlation effects were described using the Perdew-Burke-Ernzerhof generalised gradient approximation ${ }^{66}$. A kinetic energy cut-off of $400 \mathrm{eV}$ was used, with a $3 \times 3 \times 3$ Monkhorst-Pack grid for electron density k-points employed in the case with super-cell $(8 \times 8 \times 8)$ calculations $(1024+N$ atoms, with $\mathrm{N}$ up to 22 atoms) for the $1 / 2\langle 111\rangle$ and $\langle 100\rangle$ interstitial defects. The set of interstitial defect clusters used was the same as in ref ${ }^{67}$, with the difference that in the earlier work the energies reported were in the constant volume approximation employing the Varvenne et al. cell size correction ${ }^{57}$, whereas here the full cell relaxation method was used. A systematic study of fully-relaxed defect formation volumes for the $\langle 110\rangle$ self-interstitial clusters within the underlying three-dimensional C15 Laves phase structure ${ }^{68}$ was also performed. The small C15 interstitial clusters in the bcc lattice of iron are known to be highly stable in a comparison with the $1 / 2\langle 111\rangle$ selfinterstitial defect clusters due to magnetic effects. Here we include them for completeness, as their formation energy compared to interstitial loops may be affected by the applied strain ${ }^{69}$.

Randomly generated vacancy clusters were produced by removing atoms on a random path through an otherwise perfect crystal. The path was allowed to move in $\langle 111\rangle$ and $\langle 100\rangle$ directions, and allowed to overlap itself. A path of length $L$ steps leads to $\leq L$ vacancies placed in a loose cluster. These vacancy clusters were then relaxed and the lowest energy structures were passed to DFT for a comparison calculation. It is important to emphasize that in the present DFT calculations for both SIA and vacancy clusters, the full cell relaxation method has been adopted to investigate the relaxation volume of 
defect structures. Details of the DFT calculations can be found in Ref. ${ }^{46}$. Results are shown in figure 4.

The slab model including a 18 Åvacuum layer between the top and bottom surfaces has been employed for calculations of W(100), W(110), W(111) and W(211) surface energies where 15, 15, 24 and 24 atomic layers were used, respectively, to ensure DFT convergence.

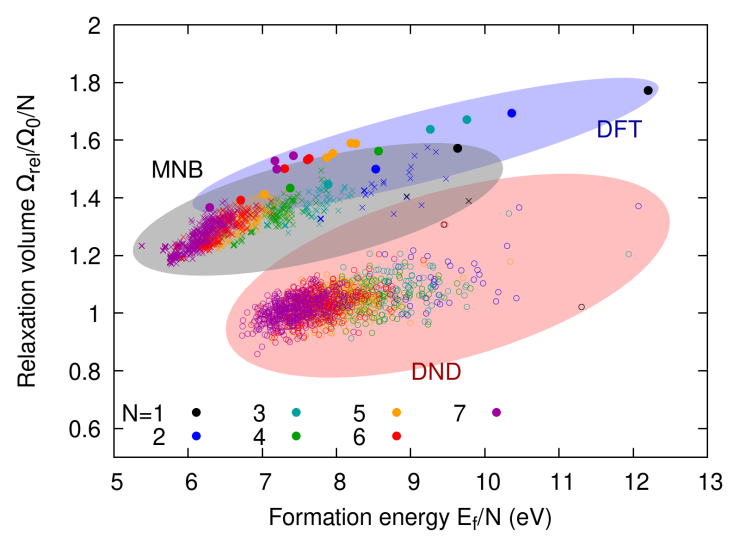

FIG. 3. Relaxation volumes of randomly generated interstitial defect clusters for different numbers of interstitial atoms in the defect, $N$. DFT values for energies and relaxation volumes from Ref. ${ }^{13}$ are shown with filled circles. Crosses: the values computed with MNB potential, open circles are the values computed using the DND potential. Shaded ellipses are drawn to guide the eye to the regions covered by data generated uisng the relevant potentials. Note that the DND potential tends to predict a higher formation energy and lower relaxation volume of a defect cluster than the MNB potential.

Two trends are immediately apparent in figures 3 and 4. Firstly, we see that larger clusters have a lower formation energy per point defect. This is just an illustration of the reduction in energy due to coalescence of individual point defects into a cluster of defects. But we also can see that the magnitude of the relaxation volume reduces with cluster size. By coalescing, the volume strain on the lattice required to accommodate small defects is reduced.

The second trend we see is that the distribution of relaxation volumes is small, of order \pm 0.1 atomic volume per point defect, despite the random nature of the defect cluster generation.

A visually striking consequence of this is that the scatter plots for different potentials do not significantly overlap, but this is of limited physical significance as the offset for each potential is a consequence of its fitting, and the fitting did not consider relaxation volume.

The true physical significance of this result is that even if the exact structure of a defect cluster is not known, its relaxation volume can often be estimated from the number of point defects that it contains. The accuracy in the estimate of the relaxation volume may be rather low, as the different potentials given different offsets, but this lack of accuracy will itself be small compared to the cur-

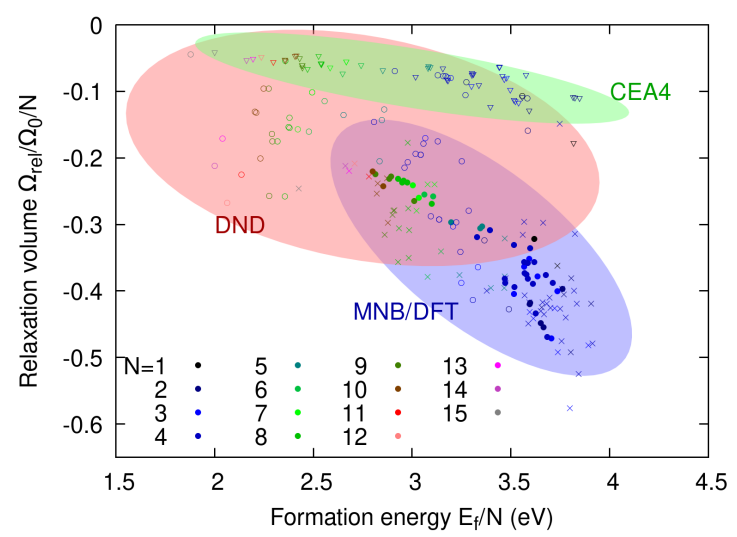

FIG. 4. Relaxation volumes of randomly generated vacancy defect clusters for different numbers of vacant sites in the defect, $N$. DFT values for energies are from Ref. ${ }^{46}$, the relaxation volumes were computed in this study, and are shown with filled circles. Crosses: the values computed with MNB potential, open circles with DND potential. Shaded ellipses are drawn to guide the eye to the regions covered by the potentials. Note that the DND potential tends to predict a lower formation energy and smaller magnitude relaxation volume than the MNB potential. The CEA potential predicts the smallest relaxation volume. The MNB potential data have a high degree of overlap with the DFT relaxation volume data.

rent accuracy in modelling the time-dependent evolution of cascades.

Tabulated values of the relaxation volumes of low energy small defect clusters are given in table III.

\section{B. Lowest energy defect structures}

Having considered randomly-generated defect clusters, we now turn our attention to larger low energy defects. Experimentally, both the $\langle 100\rangle$ and $1 / 2\langle 111\rangle$ interstitial and vacancy-type loops are observed in ion-irradiated ultra-high purity tungsten foil in the $\mathrm{TEM}^{9,70}$. We will consider these four loop types as idealised, planar, circular, prismatic loops, such as might be the basis set for time-evolution in object $\mathrm{kMC}$ or $\mathrm{CD}$. To these objects we add the spherical voids. It may be that, especially at larger sizes, the facetted voids, or hexagonal prismatic loops have slightly lower energy ${ }^{71,72}$. For our purposes it is not necessary to guarantee that we have the true ground state of a defect cluster as we are attempting to find trends that govern the variation in relaxation volume, independent to the choice of interatomic interaction potential.

We construct the prismatic loops and spherical voids using the procedure proposed in ${ }^{73}$. Formation energies and relaxation volumes for the interstitial defects are presented in figure 5 , and for vacancy defects in figure 6 . The formation energies are included here as a comparison to previous studies, and to provide a complete empirically 


\begin{tabular}{l|l|rrrrrrr} 
method & \multicolumn{8}{|c}{$\Omega_{\text {rel }} / \Omega_{0}$} \\
& character & $\mathrm{N}=1$ & 2 & 3 & 4 & 5 & 6 & 7 \\
\hline DFT & vac & -0.32 & -0.84 & -1.21 & -1.28 & -1.48 & -1.53 & -1.69 \\
MNB & vac & -0.36 & -0.85 & -1.29 & -1.60 & -1.88 & -2.27 & -1.96 \\
DND & vac & -0.11 & -0.17 & -0.51 & -0.58 & -0.68 & -0.96 & -1.10 \\
CEA4 & vac & -0.18 & -0.22 & -0.28 & -0.32 & -0.35 & -0.45 & -0.46 \\
\hline DFT & $\mathrm{i}\langle 111\rangle$ & 1.57 & 3.00 & 4.34 & 5.73 & 7.06 & 8.35 & 9.56 \\
MNB & $\mathrm{i}\langle 111\rangle$ & 1.40 & 2.65 & 3.92 & 5.08 & 6.25 & 7.39 & 8.63 \\
DND & $\mathrm{i}\langle 111\rangle$ & 1.31 & 2.41 & 3.48 & 3.85 & 4.53 & 5.76 & 6.45 \\
CEA4 & $\mathrm{i}\langle 111\rangle$ & 1.25 & 2.38 & 4.57 & 4.80 & 6.06 & 7.36 & 6.97 \\
\hline DFT & $\mathrm{i}\langle 100\rangle$ & 1.77 & 3.39 & 4.91 & 6.25 & 7.77 & 9.00 & 10.70 \\
DFT & $\mathrm{i} \mathrm{C} 15$ & & 3.92 & & 6.59 & 8.19 & 9.37 & 11.00
\end{tabular}

TABLE III. The relaxation volumes $\left(\Omega_{r e l} / \Omega_{0}\right)$ of the lowest energy vacancy and interstitial clusters containing $N$ point defects, $1 \leq N \leq 7$. These relaxation volumes were computed using full relaxation of the simulation cell and atom positions.

parameterized data set for future multiscale modelling studies. We find that our answers agree with results given in Refs. ${ }^{72,73}$ to order of the symbol size.

We can see from figure 5 that the relaxation volume of a large interstitial loop tends to $\lim _{N \rightarrow \infty} \Omega_{r e l} / \Omega_{0}=N$, where $\Omega_{0}$ is the atomic volume. This result confirms that the volume per atom in an edge dislocation, which is a semi-infinite plane of atoms embedded in a crystal lattice, must be $\Omega_{0}$. What is more surprising is the slow rate at which the result converges to this answer. Though the vertical scale in figure 5 is chosen to exaggerate the effect, nevertheless it can be that loops need to be well over one hundred point defects, perhaps even over one thousand before this limit can truly be said to be reached. To give the reader an idea of the spatial scale involved, a circular $1 / 2\langle 111\rangle$ dislocation loop containing one thousand interstitials has the diameter of $8.5 \mathrm{~nm}$. A second interesting feature of figure 5 is that the relaxation volume is not necessarily a monotonic function of the number of interstitial atoms $N$.

A regression analysis of the relaxation volume as a function of size for interstitial loops suggests that an excellent fit can be found for the empirical form

$$
\Omega_{r e l} / \Omega_{0}=N+b_{0} \sqrt{N} \ln N+b_{1} \sqrt{N}+b_{2} .
$$

We fit this using least squares fitting to $\left(\Omega_{\text {rel }} / \Omega_{0}-N\right) / \sqrt{N}$, and find error bar estimates using bootstrapping ${ }^{74}$. Fits for the relaxation volumes and formation energies are given in tables IV and $\mathrm{V}$ respectively. Formation energies have been fitted to $E_{f} / \sqrt{N}$ using the same least squares method.

In figure 6 we see that the relaxation volume of a large vacancy loop tends to $\lim _{N \rightarrow \infty} \Omega_{\text {rel }} / \Omega_{0}=-N$, and again may be non-monotonic. At small cluster sizes $N<30$, however, it is not clear if there should be a single function describing the relation between the relaxation volume and point defect count $N$. Small vacancy loops are unstable with respect to their transformation to open platelets $^{73}$ and subsequently to spherical voids, particularly for the DND and CEA-4 potentials, so the smallest relaxed vacancy clusters may not be strictly classified as 'loops'. We have omitted small vacancy clusters which show significantly different elastic properties to large loops.

A regression analysis of relaxation volume and formation energy of the C15 structures computed using DFT are tabulated in table VII. The formation energy is fitted to $E_{f}=a_{0} N+a_{1} N^{2 / 3}+a_{2}$, indicating that the energy is driven by both volume and surface energy terms. The relaxation volume is well fitted by this same form, $\Omega_{\text {rel }} / \Omega_{0}=b_{0} N+b_{1} N^{2 / 3}+b_{2}$. It is not required that the relaxation volume per $\mathrm{C} 15$ interstitial tends to $\lim _{N \rightarrow \infty} \Omega_{r e l} / \Omega_{0}=N$, as the structure is not bcc. We note that the $\mathrm{C} 15$ structures are higher energy than the $1 / 2\langle 111\rangle$ interstitial dislocation loops. Our fitting suggests that at larger defect sizes C15 will be less stable than dislocation loops.

A regression analysis of relaxation volume and formation energy of voids are tabulated in table VI. A regression analysis of the relaxation volume of a void as a function of void size suggests that an excellent fit can be found for the form $\Omega_{r e l} / \Omega_{0}=b_{0} N^{2 / 3}+b_{1}$, for $N>5$. The two-thirds power implies that the elastic relaxation of a void is driven by the minimization of the surface energy of the void, and the resulting elastic contraction of the material around it. Results for the relaxation volumes are given in table VI, and the DFT fit is shown on figure 6 . The MNB potential predictions are in excellent agreement with the DFT results for the relaxation volume. The relaxation volumes of the lowest energy vacancy clusters for $1 \leq N \leq 7$ are given in table III. We analyse the relaxation volume of the void using linear elasticity theory in section IIIB. The formation energy in table VI is fitted to $E_{f}=a_{0} N^{2 / 3}+a_{1}$, indicating that the energy is driven by surface energy alone.

\section{The anisotropy of the elastic relaxation}

In equation 4 we expressed the total relaxation volume as the sum of the three partial relaxation volumes. These three partial volumes, plus the corresponding eigenvectors, completely specify the tensor $\Omega_{k l}$, and hence the dipole tensor through eq. (3). 


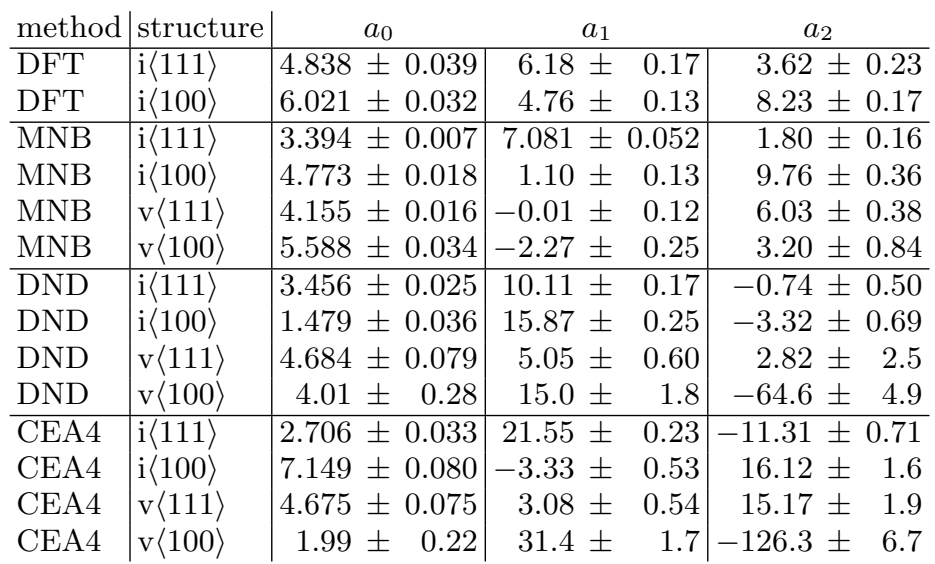

TABLE IV. Linear regression fits for the formation energy (in eV) of interstitial and vacancy loops fitted to the form $E_{f}=$ $a_{0} \sqrt{N} \ln N+a_{1} \sqrt{N}+a_{2}$. The DFT values shown are from this study using the cell relaxation method. Note that the fits should only be considered accurate in the ranges covered by the points in figures 5,6 .

\begin{tabular}{|c|c|c|c|c|}
\hline method & structure & $b_{0}$ & $b_{1}$ & $b_{2}$ \\
\hline DFT & $\mathrm{i}\langle 111\rangle$ & $-1.983 \pm 0.007$ & $2.614 \pm 0.026$ & $-3.195 \pm 0.035$ \\
\hline DFT & $\mathrm{i}\langle 100\rangle$ & $-1.977 \pm 0.003$ & $2.698 \pm 0.011$ & $-3.330 \pm 0.015$ \\
\hline $\mathrm{MNB}$ & $\mathrm{i}\langle 111\rangle$ & $0.008 \pm 0.002$ & $0.738 \pm 0.010$ & $-0.179 \pm 0.021$ \\
\hline MNB & $\mathrm{i}\langle 100\rangle$ & $-0.420 \pm 0.007$ & $0.622 \pm 0.048$ & $2.13 \pm 0.16$ \\
\hline MNB & $\mathrm{v}\langle 111\rangle$ & $0.253 \pm 0.002$ & $-0.480 \pm 0.008$ & \\
\hline MNB & $\mathrm{v}\langle 100\rangle$ & $-0.155 \pm 0.003$ & $1.053 \pm 0.015$ & \\
\hline DND & $\mathrm{i}\langle 111\rangle$ & $-0.061 \pm 0.016$ & $-1.566 \pm 0.096$ & $2.95 \pm$ \\
\hline DND & $\mathrm{i}\langle 100\rangle$ & $-1.348 \pm 0.006$ & $3.534 \pm 0.032$ & \\
\hline DND & $\mathrm{v}\langle 111\rangle$ & $0.112 \pm 0.009$ & $-5.462 \pm 0.055$ & $25.07 \pm$ \\
\hline DND & $\mathrm{v}\langle 100\rangle$ & $0.377 \pm 0.081$ & $-6.83 \pm 0.49$ & $39.43 \pm$ \\
\hline CEA4 & $\mathrm{i}\langle 111\rangle$ & $-0.050 \pm 0.018$ & $7.44 \pm$ & $-22.20 \pm$ \\
\hline CEA4 & $\mathrm{i}\langle 100\rangle$ & $0.845 \pm 0.027$ & $-0.94 \pm$ & $1.60 \pm$ \\
\hline CEA4 & $\mathrm{v}\langle 111\rangle$ & $0.384 \pm 0.015$ & $-0.19 \pm$ & $2.08 \pm$ \\
\hline CEA4 & $\mathrm{v}\langle 100\rangle$ & $0.554 \pm 0.041$ & $-0.14 \pm$ & $13.9 \pm$ \\
\hline
\end{tabular}

TABLE V. Linear regression fits for the relaxation volume $\left(\Omega_{r e l} / \Omega_{0}\right)$ of interstitial and vacancy loops fitted to the form $\Omega_{r e l} / \Omega_{0}= \pm N+b_{0} \sqrt{N} \ln N+b_{1} \sqrt{N}+b_{2}$, where the positive and negative signs are for interstitial and vacancy loops respectively. Note that the fits should only be considered accurate in the ranges covered by the points in figures 5,6 .

\begin{tabular}{|c|c|c|c|c|}
\hline method & $a_{0}$ & $a_{1}$ & $b_{0}$ & $b_{1}$ \\
\hline $\mathrm{DFT}$ & $7.18 \pm 0.05$ & $-7.72 \pm 0.16$ & $-0.49 \pm$ & $-0.23 \pm 0.13$ \\
\hline MNB & $7.35 \pm 0.06$ & $-7.4 \pm 3.0$ & $-0.50 \pm$ & $-0.77 \pm 0.40$ \\
\hline DND & $5.25 \pm 0.05$ & $-1.9 \pm$ & $-0.31 \pm$ & $0.3 \pm 1.1$ \\
\hline CEA4 & $5.56 \pm 0.06$ & $-3.2 \pm$ & $-0.122 \pm 0.003$ & $0.10 \pm 0.12$ \\
\hline
\end{tabular}

TABLE VI. Linear regression fits for the formation energy (in eV) and relaxation volume $\Omega_{\text {rel }} / \Omega_{0}$ of vacancy clusters and voids. The relaxation volume is fitted to the form $\Omega_{r e l} / \Omega_{0}=b_{0} N^{2 / 3}+b_{1}$, indicating this too is driven by surface energy. The DFT data use the lowest energy structures for $1 \leq N \leq 10$, the EAM data use the lowest energy structures for $6 \leq N \leq 10$ and spherical voids for $15 \leq N \leq 1067$. The energy is fitted to the form $E_{f}=a_{0} N^{2 / 3}+a_{1}$, indicating a domination by surface energy.

\begin{tabular}{l|ccc|ccc} 
method & $a_{0}$ & $a_{1}$ & $a_{2}$ & $b_{0}$ & $b_{1}$ & $b_{2}$ \\
\hline DFT & $2.37 \pm 0.06$ & $7.39 \pm 0.20$ & $4.92 \pm 0.29$ & $1.22 \pm 0.01$ & $0.39 \pm 0.03$ & $0.87 \pm 0.04$
\end{tabular}

TABLE VII. Linear regression fits for the formation energy (in eV) and relaxation volume $\Omega_{r e l} / \Omega_{0}$ of interstitial clusters in the C15 structure. Relaxation volume are fitted to $\Omega_{r e l} / \Omega_{0}=b_{0} N+b_{1} N^{2 / 3}+b_{2}$. The structures taken were those used in ref ${ }^{67}$, using the cell relaxation method, with sizes $2 \leq N \leq 22$. Energies are fitted to $E_{f}=a_{0} N+a_{1} N^{2 / 3}+a_{2}$, as suggested by ref ${ }^{67}$, indicating terms dependent on volume and surface area. 


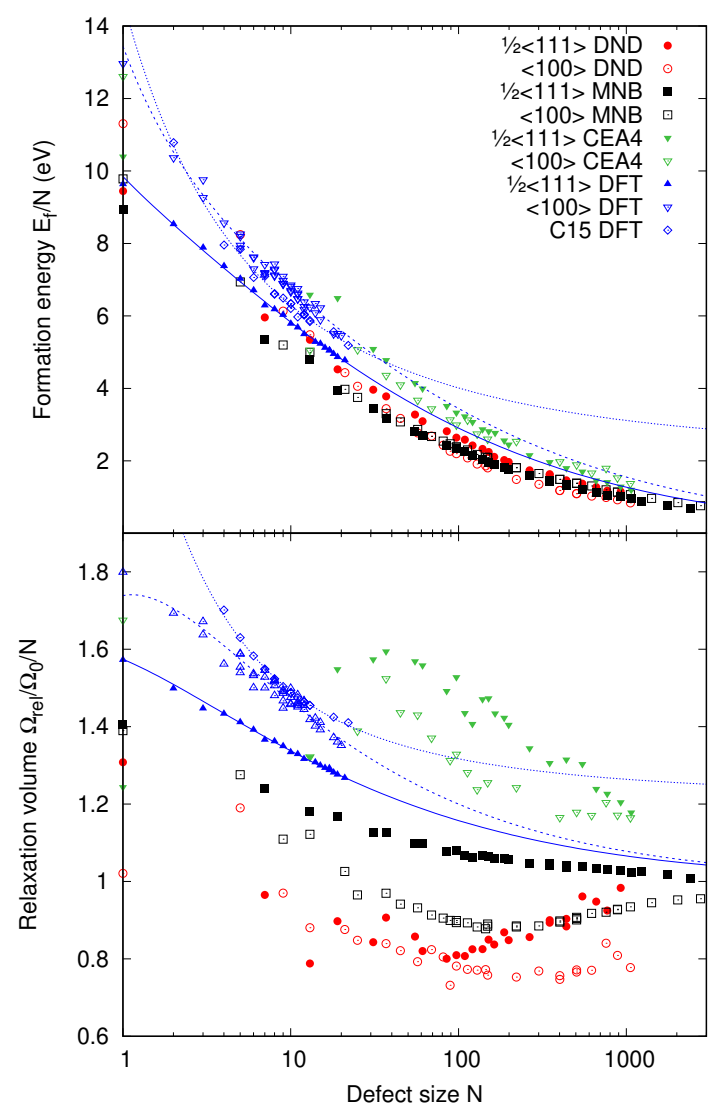

FIG. 5. Formation energy and relaxation volume of low energy interstitial defect clusters. All clusters and loops are of circular shape. DFT values for formation energies are extrapolated with lines fitted to $E_{f}=a_{0} \sqrt{N} \ln N+a_{1} \sqrt{N}+a_{2}$ (see table IV). DFT values for relaxation volumes are extrapolated with lines fitted to $\left|\Omega_{r e l} / \Omega_{0}\right|=N+b_{0} \sqrt{N} \ln N+$ $b_{1} \sqrt{N}+b_{2}$ ( see table $\mathrm{V}$ ). Note that the energies for ideal interstitial defects computed with the potentials are very similar, but the relaxation volumes differ considerably, with the DND potential typically predicting smaller values and CEA4 larger.

For cubic crystals, it is straightforward to invert the matrix equations and recover the dipole tensor. For the perfect defect cluster shapes considered in section II B, one eigenvector describes both the Burgers vector and normal of the loops, and so this task can be accomplished simply and analytically.

For a $\mathbf{b}=1 / 2[111]$ loop with the $\{111\}$ habit plane (as is the lowest energy case in tungsten), the dipole tensor has the general form

$$
P_{1 / 2[111]}=\left(\begin{array}{ccc}
a & b & b \\
b & a & b \\
b & b & a
\end{array}\right),
$$

where $a$ and $b$ are numerical parameters with units of energy. The dipole tensor for other symmetry related $1 / 2\langle 111\rangle$ loops is found by taking negative signs in the offdiagonal elements as appropriate. In a cubic crystal, we

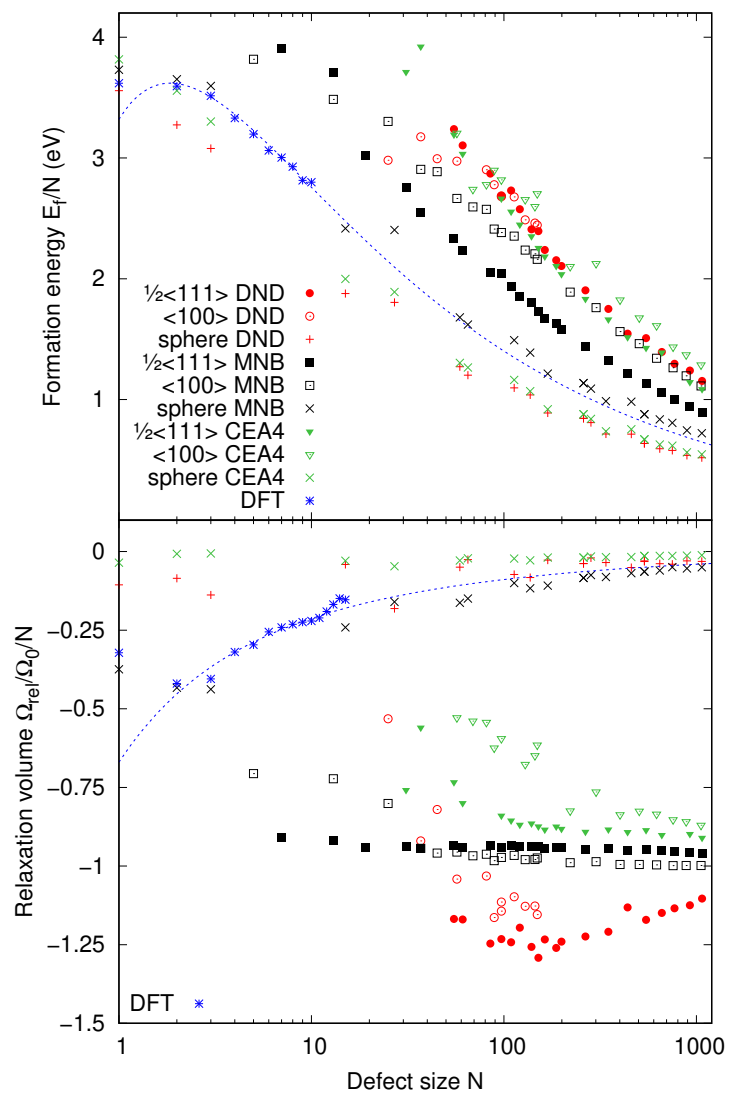

FIG. 6. Formation energy and relaxation volumes of low energy vacancy defect clusters. The loops were generated with circular shapes, and the voids as spheres. DFT computed energies of the low-energy vacancy clusters from $\operatorname{Ref}^{46}$, with an extrapolated line fitted to $E_{f}=a_{0} N^{2 / 3}+a_{1}$. DFT computed relaxation volumes for the same structures in $\operatorname{Ref}^{46}$ fitted to $\Omega_{r e l} / \Omega_{0}=b_{0} N^{2 / 3}+b_{1}$.

can readily find the elastic compliance tensor $\left(S \equiv C^{-1}\right)$. Its representation as a matrix in Voigt notation has the simple form:

$$
S=\left(\begin{array}{cccccc}
\frac{c_{11}+c_{12}}{d} & \frac{-c_{12}}{d} & \frac{-c_{12}}{d} & 0 & 0 & 0 \\
\frac{-c_{12}}{d} & \frac{c_{11}+c_{12}}{d} & \frac{-c_{12}}{d} & 0 & 0 & 0 \\
\frac{-c_{12}}{d} & \frac{-c_{12}}{d} & \frac{c_{11}+c_{12}}{d} & 0 & 0 & 0 \\
0 & 0 & 0 & \frac{1}{c_{44}} & 0 & 0 \\
0 & 0 & 0 & 0 & \frac{1}{c_{44}} & 0 \\
0 & 0 & 0 & 0 & 0 & \frac{1}{c_{44}}
\end{array}\right)
$$

with $d=c_{11}^{2}+c_{11} c_{12}-2 c_{12}^{2}$, and hence, using equation 3 for tensor $\Omega_{k l}$, we find the partial relaxation volumes

$$
\begin{aligned}
\Omega^{(1)}=\Omega^{(2)} & =\frac{a}{c_{11}+2 c_{12}}-\frac{b}{c_{44}} \\
\Omega^{(3)} & =\frac{a}{c_{11}+2 c_{12}}+2 \frac{b}{c_{44}} .
\end{aligned}
$$

We can define a single dimensionless measure of the anisotropy of relaxation for the structures considered 
here as a ratio of the smallest to largest (magnitude) partial relaxation volumes,

$$
\alpha \equiv \frac{\Omega^{(1)}}{\Omega^{(3)}} .
$$

The value $\alpha=1$ indicates that all the three partial relaxation volumes are equal, as should be the case for a spherical void. A value in the interval $0<\alpha<1$ indicates that the principal lattice relaxation is along the Burgers vector, but there are also smaller relaxations in the two orthogonal directions with the same sign (i.e. compressive or tensile ) as the principal relaxation. The value $\alpha=0$ indicates that the only lattice relaxation is along the Burgers vector. A value $\alpha<0$ indicates that the principal lattice relaxation is along the Burgers vector, but there are also smaller relaxations in the two orthogonal directions with the opposite sign (i.e. compressive or tensile ) as the principal relaxation.

For a $1 / 2\langle 111\rangle$ loop, the anisotropy parameter is

$$
\alpha_{1 / 2}\langle 111\rangle=\frac{c_{44} a-\left(c_{11}+2 c_{12}\right) b}{c_{44} a+2\left(c_{11}+2 c_{12}\right) b} .
$$

Given the value of $\alpha$ and the total relaxation volume $\Omega_{\text {rel }}$ we can reconstruct the dipole tensor for a $1 / 2[111]$ loop as

$$
\begin{aligned}
& P_{11}=P_{22}=P_{33}=a=\frac{c_{11}+2 c_{12}}{3} \Omega_{r e l} \\
& P_{12}=P_{23}=P_{31}=b=\frac{c_{44}(\alpha-1)}{3(1+2 \alpha)} \Omega_{r e l}
\end{aligned}
$$

The same process can be followed for a [001] loop, for which the dipole tensor is

$$
P_{[001]}=\left(\begin{array}{ccc}
a & 0 & 0 \\
0 & a & 0 \\
0 & 0 & a^{\prime}
\end{array}\right) .
$$

This gives partial relaxation volumes

$$
\begin{aligned}
\Omega^{(1)}=\Omega^{(2)} & =\frac{c_{11} a}{d}-\frac{c_{12} a^{\prime}}{d} \\
\Omega^{(3)} & =-\frac{2 c_{12} a}{d}+\frac{\left(c_{11}+c_{12}\right) a^{\prime}}{d},
\end{aligned}
$$

and the anisotropy coefficient

$$
\alpha_{\langle 100\rangle}=\frac{c_{11} a-c_{12} a^{\prime}}{-2 c_{12} a+\left(c_{11}+c_{12}\right) a^{\prime}} .
$$

The parameter $d$ is defined above. Given $\alpha$ and the total relaxation volume $\Omega_{r e l}$, we can also reconstruct the dipole tensor for a [001] loop as

$$
\begin{gathered}
P_{11}=P_{22}=a=d \frac{c_{12}(\alpha-1)+c_{11} \alpha}{\left(c_{11}^{2}+c_{11} c_{12}+2 c_{12}^{2}\right)(1+2 \alpha)} \Omega_{r e l} \\
P_{33}=a^{\prime}=d \frac{c_{11}+2 c_{12} \alpha}{\left(c_{11}^{2}+c_{11} c_{12}+2 c_{12}^{2}\right)(1+2 \alpha)} \Omega_{r e l} \\
P_{12}=P_{23}=P_{31}=0
\end{gathered}
$$

For spherical voids the coefficient $\alpha_{\text {sphere }}=1$. The dipole tensor for a spherical void can be reconstructed $\mathrm{as}^{20}$

$$
\begin{aligned}
& P_{11}=P_{22}=P_{33}=\frac{c_{11}+2 c_{12}}{3} \Omega_{r e l}=K \Omega_{r e l} \\
& P_{12}=P_{23}=P_{31}=0,
\end{aligned}
$$

where $K$ is the bulk modulus.

We choose to present a parameterization required to fully reconstruct the dipole tensor as the pair of values $\left\{\Omega_{r e l}, \alpha\right\}$. The latter is plotted in figure 7 . Fits to this data are given in table VIII.

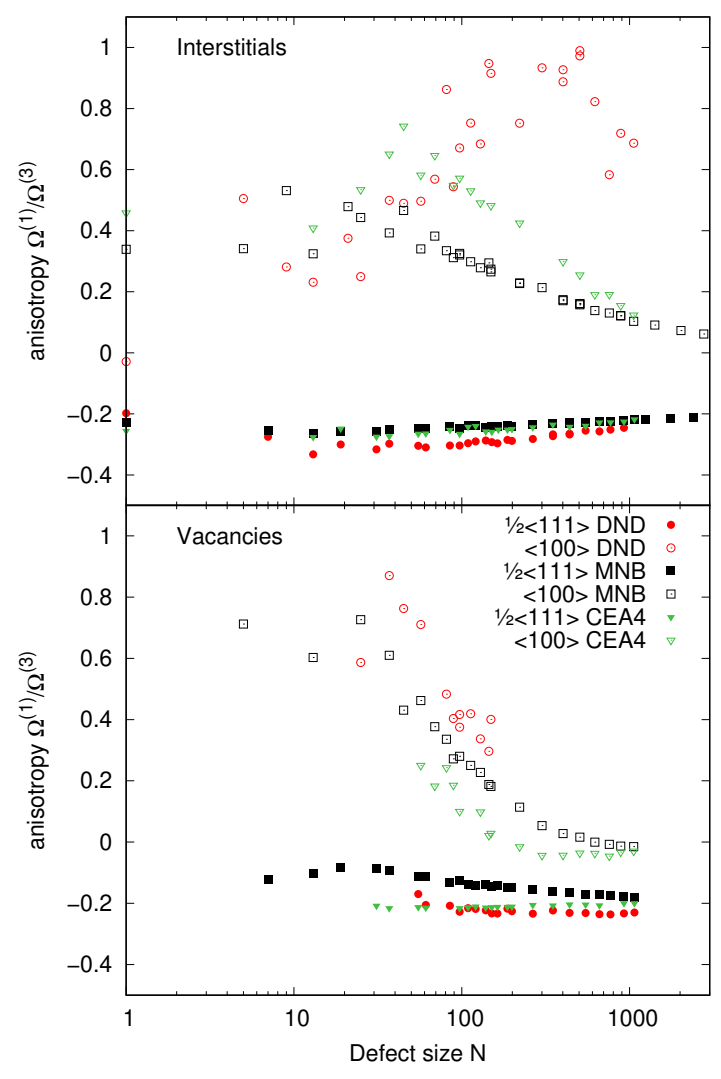

FIG. 7. The relaxation volume anisotropy $\alpha$, as defined by the ratio of the smallest to the largest partial relaxation volume (see equation 10), for idealised circular prismatic loops.

\section{Cascade simulations}

The final set of configurations we consider are taken from the MD cascade simulations dataset provided for the IAEA Visualisation Challenge ${ }^{75}$. The simulations were evolved using a stiffened DND potential ${ }^{76}$ until a simulated time 40ps. The simulation cell was initially a perfect crystal, with damping applied to atoms with kinetic energy over $10 \mathrm{eV}^{77}$, and with an additional thermostat at the boundaries of the supercell ${ }^{78}$. One atom 


\begin{tabular}{|c|c|c|c|c|c|}
\hline methe & & $\alpha_{0}$ & $\alpha_{1}$ & $\alpha_{2}$ & $\alpha_{3}$ \\
\hline MNB & $\mathrm{i}\langle 111\rangle$ & -0.195 & $-0.144 \pm 0.001$ & $0.189 \pm 0.006$ & $-0.212 \pm 0.018$ \\
\hline MNB & $\mathrm{i}\langle 100\rangle$ & 0 & $0.196 \pm 0.007$ & $2.320 \pm 0.050$ & $-2.47 \pm 0.14$ \\
\hline MNB & $\mathrm{v}\langle 111\rangle$ & -0.195 & $0.020 \pm 0.003$ & $0.514 \pm 0.017$ & $-0.331 \pm 0.044$ \\
\hline MNB & $\mathrm{v}\langle 100\rangle$ & 0 & $0.567 \pm 0.041$ & $-3.16 \pm 0.25$ & $19.78 \pm 0.66$ \\
\hline DND & $i\langle 111\rangle$ & -0.203 & $-0.255 \pm 0.004$ & $0.270 \pm 0.030$ & $-0.216 \pm 0.089$ \\
\hline DND & $\mathrm{i}\langle 100\rangle$ & & $5.826 \pm 0.075$ & $-19.51 \pm 0.47$ & $17.6 \pm$ \\
\hline DND & $\mathrm{v}\langle 111\rangle$ & -0.203 & $-0.226 \pm 0.004$ & $0.557 \pm 0.029$ & $3.67 \pm$ \\
\hline DND & $\mathrm{v}\langle 100\rangle$ & 0 & $-0.254 \pm 0.064$ & $5.50 \pm 0.36$ & $-0.9 \pm \quad 1.3$ \\
\hline CEA4 & $i\langle 111\rangle$ & -0.199 & $-0.128 \pm 0.004$ & $0.036 \pm 0.028$ & $-0.069 \pm 0.081$ \\
\hline CEA4 & $\mathrm{i}\langle 100\rangle$ & 0 & $0.157 \pm 0.033$ & $4.57 \pm 0.21$ & $-4.63 \pm 0.56$ \\
\hline CEA4 & $\mathrm{v}\langle 111\rangle$ & -0.199 & $-0.028 \pm 0.002$ & $0.103 \pm 0.012$ & $-0.961 \pm 0.030$ \\
\hline CEA4 & $\mathrm{v}\langle 100\rangle$ & 0 & $0.091 \pm 0.023$ & $-3.01 \pm 0.16$ & $36.8 \pm 0.51$ \\
\hline
\end{tabular}

TABLE VIII. The dimensionless relaxation volume anisotropy parameter $\alpha$, as defined by the ratio of the smallest to the largest partial relaxation volume ( see equation 10 ), for idealised circular prismatic loops, fitted to $\alpha=\alpha_{0}+\alpha_{1} \ln N / \sqrt{N}+\alpha_{2} / \sqrt{N}+$ $\alpha_{3} / N$. The constant term $\alpha_{0}$ is derived using linear elasticity (equations 11 and 15), using the computed values for elastic constants ( see table II ).

was given an initial energy of 50-150keV, representing a primary knock-on event, and the final temperature was under $1 \mathrm{~K}$. A full description of the methodology is detailed in Refs. ${ }^{6,41}$. The cascade configurations were relaxed at constant volume using the procedure detailed in Ref. ${ }^{13}$ with different EAM potentials, and the relaxation volume computed using the stress method.

The results for the energy and relaxation volume as a function of the number of Frenkel pairs produced are shown in figure 8 . We see that there is a slight tendency for a lower energy per Frenkel pair for the largest cascades. This may indicate that the largest cascades (in terms of Frenkel pairs produced ) are associated with the largest dislocation loops, and the largest loops have the lowest formation energy per point defect. The relaxation volume shows a clear correlation with the Frenkel pair count, but for individual cascades there can be considerable variation. This is consistent with the preceding results, if the defect clusters are weakly interacting, as the relaxation volume should be determined by the degree of clustering rather than the total number of defects. On the basis of these simulations, it is reasonable to give a single relaxation volume per cascade. We find from the relaxed cascades

$$
\Omega_{r e l} / \Omega_{0}=b_{0} N_{F P},
$$

with $b_{0}=0.77 \pm 0.01$ for MNB and $b_{0}=0.50 \pm 0.02$ for DND.

Finally we can establish the predictive quality of the tabulated data presented here by using tables III,V,VI to estimate the relaxation volume of a cascade configuration. After the initial Wigner-Seitz analysis of the cascade configuration, clusters of interstitials and vacancies were grouped where pairs of like-character point defects were separated by nearest- or next-nearest neighbours. As many clusters are too small to perform a DXA analysis $^{79}$, we assert that all the interstitial loops are of $1 / 2\langle 111\rangle$ type. The relaxation volume for each defect is then looked up, and the total volume summed. The result is displayed in figure 9. While there is some scatter for MNB potential, and possibly additional relaxation within the cascade for the DND potential, it is clear that the simple empirical fits for mesoscale defect relaxation volume give a reasonable estimate of the cascade relaxation volume. This is an important result for the transferability of our approach. It indicates that even in the extreme case of high-energy defects formed close together in a cascade, the interaction between defects has a small effect on their relaxation volumes, and so elastically at least the defects can be treated as quasi-independent. This therefore demonstrates that the relaxation volume is a 'good' phase field for multiscale modelling, in the sense that it is additive with respect to the concentration of defects in the underlying microstructure.

\section{DISCUSSION OF RESULTS}

\section{A. A comparison with isotropic linear elasticity}

In section IIC we defined the anisotropy parameter in the partial relaxation volumes of idealised loop defects considered in section II B. The expected values of the anisotropy parameter can be computed using linear elasticity as follows. The dipole tensor for a dislocation loop with normal $\hat{\mathbf{n}}$ and Burgers vector $\mathbf{b}$ and area $\mathrm{A}$ in isotropic elasticity is given by ${ }^{20,80}$

$$
P_{i j}=A\left[\mu\left(b_{i} n_{j}+b_{j} n_{i}\right)+\lambda b_{k} n_{k} \delta_{i j}\right],
$$

where $\mu=\left(c_{11}-c_{12}\right) / 2$ and $\lambda=c_{12}$ are the shear modulus and Lamé constant. Einstein summation over repeated indices is assumed. If the normal and Burgers vector are parallel, as is the case here, this expression simplifies to

$$
P_{i j}= \pm N \Omega_{0}\left[2 \mu n_{i} n_{j}+\lambda \delta_{i j}\right],
$$

where the sign is positive for the interstitial loop and negative for a vacancy loop. For the $1 / 2[111]$ loop this 

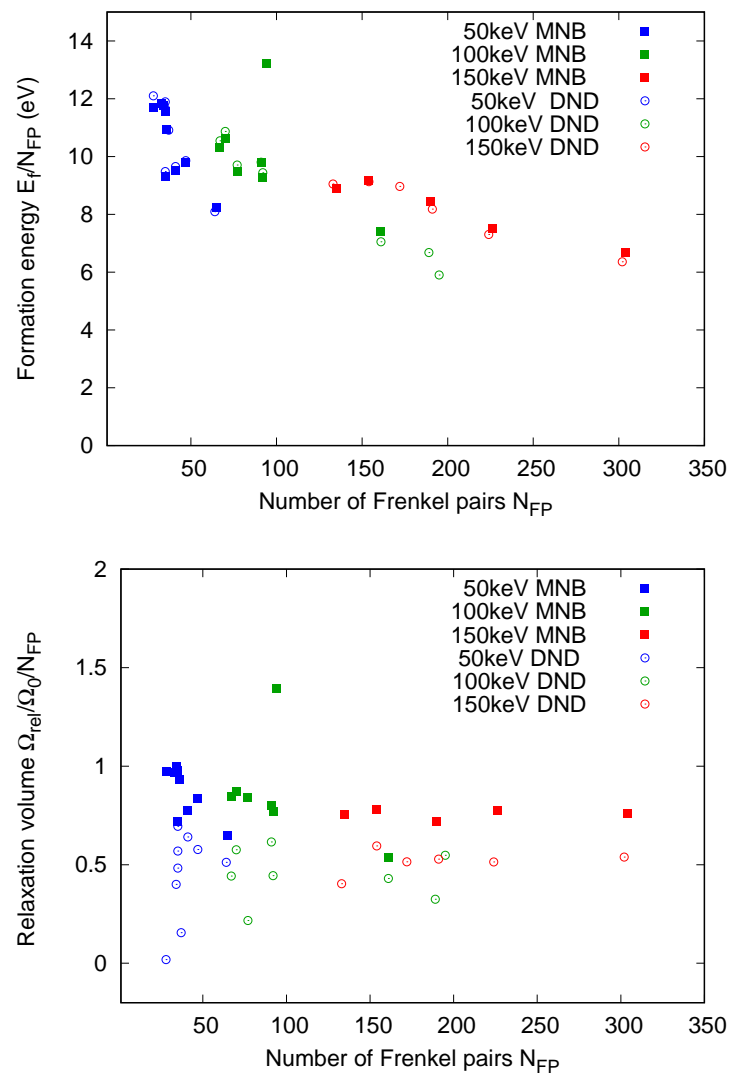

FIG. 8. Formation energy and relaxation volumes of defect clusters generated by MD cascade simulations. Filled symbols: computed with MNB potential, open circles with DND potential. The formation energy of the cascades computed with the two potentials is very similar, but MNB tends to produce a larger relaxation volume. Note that the cascade configurations were generated with the DND potential, then relaxed with both MNB and DND. Note the wide range of number of Frenkel pairs generated in a single cascade, a characteristic of the stochastic process of loop generation ${ }^{6}$, has recently been confirmed experimentally ${ }^{43}$.

reduces further to

$$
\begin{aligned}
& P_{11}=P_{22}=P_{33}= \pm K N \Omega_{0} \\
& P_{12}=P_{23}=P_{31}= \pm \frac{2 \mu}{3} N \Omega_{0}
\end{aligned}
$$

and so, substituting into equation 9 , we can identify the relaxation volume for a $1 / 2\langle 111\rangle$ loop in isotropic linear elasticity as $\Omega_{r e l}= \pm N \Omega_{0}$ and its anisotropy as $\alpha=$ $\left(-c_{11}+c_{12}+c_{44}\right) /\left(2 c_{11}-2 c_{12}+c_{44}\right)=-1 / 5$. This negative value is significant, as it indicates that for a $1 / 2\langle 111\rangle$ interstitial loop the lattice expansion is negative in directions orthogonal to the Burgers vector. Values of $\alpha$ computed using computed elastic constants are given in table VIII. Note that all the potentials give $\alpha \sim-0.2$.

Similarly, for a $1 / 2[001]$ loop we find $P_{11}=P_{22}=$ $\pm \lambda N \Omega_{0}, P_{33}= \pm(2 \mu+\lambda) N \Omega_{0}$, and $P_{12}=P_{23}=P_{31}=0$, and so the relaxation volume computed for a $\langle 100\rangle$ loop

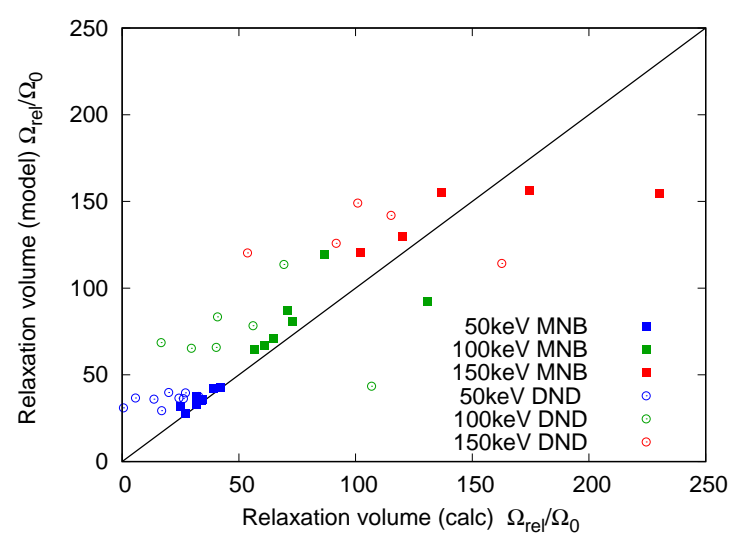

FIG. 9. A comparison of the relaxation volume computed using a full relaxation of the cascade ( $\mathrm{x}$-axis) to the relaxation volume predicted using the tabulated fits to the data ( tables III,V,VI ). The diagonal line indicates a 1:1 match- ie a perfect reproduction of the relaxation volume.

in isotropic linear elasticity is also $\Omega_{r e l}= \pm N \Omega_{0}$ but its anisotropy is $\alpha=0$.

\section{B. Relaxation volume of a vacancy cluster}

In this subsection, we derive an analytical formula for the relaxation volume of a mesoscopic spherical vacancy cluster, treating the problem in the linear elasticity approximation. The approach that we adopt here broadly follows the analysis by Wolfer and Ashkin ${ }^{81}$.

The elastic displacement field around a spherical vacancy cluster, taken in the isotropic elasticity approximation, is

$$
\mathbf{u}(\mathbf{r})=\frac{C}{r^{2}} \mathbf{n}+D r \mathbf{n},
$$

where $C$ and $D$ are constant factors that we will derive from boundary conditions, and $\mathbf{n}=\mathbf{r} / r$. The strain and stress fields associated with the spherical vacancy cluster $\operatorname{are}^{13,81}$

$$
\begin{array}{r}
\epsilon_{i j}(\mathbf{r})=\frac{C}{r^{2}}\left(\delta_{i j}-3 n_{i} n_{j}\right)+D \delta_{i j}, \\
\sigma_{i j}(\mathbf{r})=2 \mu \frac{C}{r^{2}}\left(\delta_{i j}-3 n_{i} n_{j}\right)+3 K D \delta_{i j},
\end{array}
$$

where, as above, $\mu$ is the shear modulus of the material, and $K$ is the bulk modulus.

We now find the relaxation volume of a spherical vacancy cluster of radius $a$ embedded in a concentric spherical isotropic elastic medium of radius $R$. Boundary conditions for surface tractions at the surface of the vacancy cluster $(r=a)$ and at the outer surface $(r=R)$ have the 
form

$$
\begin{gathered}
\left.\sigma_{i j} n_{j}\right|_{r=a}=-4 \mu \frac{C}{r^{3}} n_{i}+\left.3 K D n_{i}\right|_{r=a}=-p_{a}+\frac{2 s}{a}, \\
\left.\sigma_{i j} n_{j}\right|_{r=R}=-4 \mu \frac{C}{r^{3}} n_{i}+\left.3 K D n_{i}\right|_{r=R}=-p_{R}-\frac{2 s}{R},
\end{gathered}
$$

where $p_{a}$ is the pressure of gas accumulated inside the vacancy cluster (for example helium or hydrogen), $p_{R}$ the external pressure, and $s$ is the orientation-average surface stress. The surface stress $s_{i j}$ is a tensor quantity related to the surface energy by the Shuttleworth relation $s_{i j}=\gamma \delta_{i j}+\partial \gamma / \partial \epsilon_{i j}$, which can be readily computed in atomistic simulation as $s_{i j}=(1 / A) \partial(A \gamma) / \partial \epsilon_{i j}$, see $\operatorname{Re}^{82}$. This will have two non-zero eigenvalues which may be computed for each surface orientation. Here we assume an orientation average, $s$ will give a good approximation for the total relaxation volume. From the boundary conditions we find

$$
\begin{gathered}
C=\frac{a^{3} R^{3}\left(\left(p_{a}-\frac{2 s}{a}\right)-\left(p_{R}+\frac{2 s}{R}\right)\right)}{4 \mu\left(R^{3}-a^{3}\right)} \\
D=\frac{a^{3}\left(p_{a}-\frac{2 s}{a}\right)-R^{3}\left(p_{R}+\frac{2 s}{R}\right)}{3 K\left(R^{3}-a^{3}\right)},
\end{gathered}
$$

which in the limit of zero external pressure $\left(p_{R}=0\right)$ and $R \gg a$ simplify to

$$
\begin{array}{rl}
\lim _{p_{R}=0, R \gg a} C & =\frac{a^{3}}{4 \mu}\left(p_{a}-\frac{2 s}{a}\right) \\
\lim _{p_{R}}=0, R \gg a & D=0 .
\end{array}
$$

Substituting the coefficients (equation 25) back into equation 22 gives the magnitude of the displacement at the outer surface $u(R)$, and hence the change in volume.

$$
\Omega_{r e l}=\frac{4 \pi}{3}(R+u(R))^{3}-\frac{4 \pi}{3} R^{3}
$$

After some rearrangement we find

$$
\begin{aligned}
\Omega_{r e l}= & \frac{-4 \pi R^{3}}{3(3 K)^{3}}\left(p_{R}+\frac{2 s}{R}\right) \\
& \times\left(\left(p_{R}+\frac{2 s}{R}\right)^{2}-9 K\left(p_{R}+\frac{2 s}{R}\right)+27 K^{2}\right) \\
- & \frac{\pi a^{3}}{\mu(3 K)^{3}}\left(\left(p_{R}+\frac{2 s}{R}\right)-\left(p_{a}-\frac{2 s}{a}\right)\right) \\
& \times\left(p_{R}+\frac{2 s}{R}-3 K\right)^{2}(3 K+4 \mu)
\end{aligned}
$$

The first term is the response of the outer surface to the external pressure and its surface energy, independent of the presence of the void in the interior. The second term is the relaxation volume due to the void,

$$
\begin{aligned}
\Omega_{r e l}= & -\frac{\pi a^{3}}{\mu(3 K)^{3}}\left(\left(p_{R}+\frac{2 s}{R}\right)-\left(p_{a}-\frac{2 s}{a}\right)\right) \\
& \times\left(p_{R}+\frac{2 s}{R}-3 K\right)^{2}(3 K+4 \mu)
\end{aligned}
$$

In the limits $p_{R}=0$ and $R \gg a$, this simplifies to an expression for the relaxation volume of a spherical void in an elastic medium, which is filled with gas at pressure $p_{a}$

$$
\begin{aligned}
\lim _{p_{R}=0, R \gg a} \Omega_{r e l} & =\frac{\pi a^{3}\left(p_{a}-2 s / a\right)(3 K+4 \mu)}{3 K \mu} \\
& =\frac{3 \pi a^{3}}{\mu}\left(\frac{1-\nu}{1+\nu}\right)\left(p_{a}-\frac{2 s}{a}\right),
\end{aligned}
$$

where $\nu=c_{12} /\left(c_{11}+c_{12}\right)=\lambda /(2 \lambda+2 \mu)$ is Poisson's ratio.

If the internal pressure in the vacancy cluster is zero $\left(p_{a}=0\right)$, or in other words if there in no helium or hydrogen gas inside the vacancy cluster, the relaxation volume is negative and is proportional to the surface area of the cluster

$$
\Omega_{r e l} \simeq-6 \pi\left(\frac{1-\nu}{1+\nu}\right) \frac{s a^{2}}{\mu} .
$$

The radius of a spherical void can be related to the number of vacancies $N$ it contains,

$$
N \Omega_{0}=\frac{4 \pi a^{3}}{3}
$$

where $\Omega_{0}=\mathrm{a}_{0}^{3} / 2$ is the volume per atom for a bcc metal, given the lattice constant $\mathrm{a}_{0}$, so we could also write

$$
\Omega_{r e l} \simeq-\left(\frac{243 \pi}{8}\right)^{1 / 3}\left(\frac{1-\nu}{1+\nu}\right) \frac{s \mathrm{a}_{0}^{2}}{\mu} N^{2 / 3}
$$

From this equation it follows that the relaxation volume per vacancy in a vacancy cluster (a void containing no gas) is negative and varies, as the average surface stress, as the inverse third power of the number of vacancies contained in the cluster

$$
\frac{\Omega_{r e l}}{N \Omega_{0}} \sim-s N^{-1 / 3} .
$$

As a result, the relaxation volume of a void per vacancy vanishes in the macroscopic limit $N \gg 1$.

We can compare the relaxation volumes computed using atomistic relaxations to the predictions from the above surface energy model, by substituting the elastic constants and spherically-averaged surface energies $\gamma$ and stresses $s$, as computed in section A and tabulated in II. If we use a similar surface model for the formation energy, 
we find the formation energy per vacancy varies as the average surface energy and the the inverse third power of the number of vacancies that it contains

$$
\begin{aligned}
E_{f} & \simeq 4 \pi \gamma a^{2} \\
& \simeq(9 \pi)^{1 / 3} \gamma \mathrm{a}_{0}^{2} N^{2 / 3} \\
E_{f} / N & \sim \gamma N^{-1 / 3} .
\end{aligned}
$$

The comparison is shown in table IX. We conclude that the relaxation volume and formation energy of voids in tungsten are well reproduced by a simple surface model.

\section{A comparison of the empirical potentials used}

It is instructive to consider why two potentials which give very similar energies of formation for lattice defects nevertheless give quite different elastic properties. As shown in equation 5 , the dipole tensor depends on the first derivative of the potential, and for the pairwise part $V\left(r_{a b}\right)$ at least this is straightforward to analyse. The MNB potential descends from the smooth AcklandThetford form ${ }^{47,48}$, whereas the DND potential is based on a piecewise cubic spline form. This latter form has a continuous second derivative, but discontinuous third, leading to cusps in the second derivative. In the case of the DND potential, this second derivative swings from large positive to negative values. This in turn means that small changes in relative atom positions leads to large changes in the forces on the atoms. The MNB potential, by contrast, has a fairly flat first derivative. The CEA-4 potential ${ }^{44}$, which is also a cubic-spline form, but included fitting to forces during its construction, shares this flat first derivative for near equilibrium atom separations, but with more structure for greatly distorted structures. This is illustrated in figure 10.

\section{Estimating the relaxation volume per collision cascade}

In section II D we found that the relaxation volume per cascade is proportional to the number of Frenkel pairs contained. We can make an estimate for the relaxation volume per cascade if we make the assumption that the lattice defects are isolated and idealised. Sand et $\mathrm{al}^{6}$ suggest that clusters should be produced in cascades with a frequency given by the power-law

$$
f(N)=A / N^{s},
$$

and in $\mathrm{ref}^{85}$ give exponents for large clusters for interstitial-type and vacancy clusters in bulk tungsten cascade simulations as $s_{I}=1.6$ and $s_{V}=2.0$ respectively. The expected relaxation volume per Frenkel pair is then

$$
\Omega_{r e l} / N_{F P}=\sum_{N=1}^{N_{\max }} q_{I}(N) \Omega_{I}(N)+q_{V}(N) \Omega_{V}(N)
$$

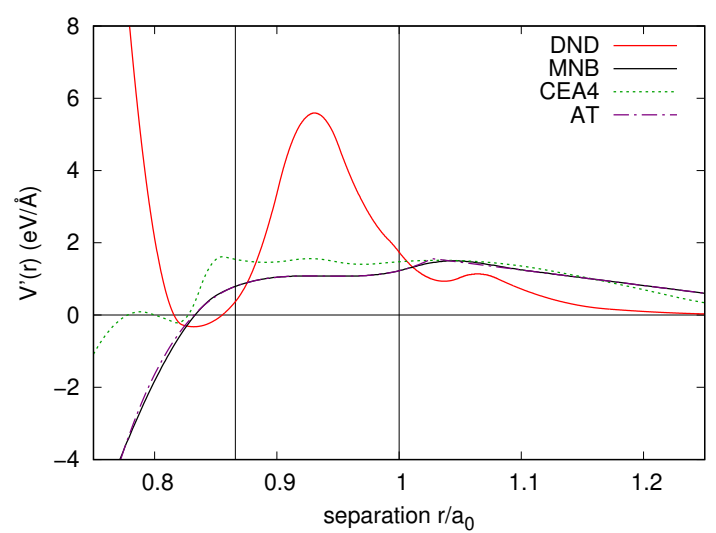

FIG. 10. The first derivative of the pairwise part of selected empirical potentials, in the effective gauge ${ }^{47,83}$ where $\rho_{e q}=$ $1, F[0]=F[1]=0$. The MNB potential is descended from the smooth Ackland-Thetford form ${ }^{47,48}$, whereas the DND potential is a piecewise cubic spline which did not consider the first derivative during fitting. Vertical lines are drawn at first and second nearest neighbour positions. Note that the DND potential is not unstable at short separation, as might be inferred from this plot, as it is stabilised by its many-body part. In MD simulations the ZBL correction is also generally applied at short range ${ }^{76,84}$. The Ackland-Thetford pairwise potential is very similar to the MNB.

with $q_{I / V}(N)$ being the weighting for an interstitial/vacancy cluster containing $N$ point defects,

$$
q_{I}(N)=\frac{N^{-S_{I}}}{\sum_{N=1}^{N_{\max }} N^{1-S_{I}}} \quad, \quad q_{V}(N)=\frac{N^{-S_{V}}}{\sum_{N=1}^{N_{\max }} N^{1-S_{V}}} .
$$

Note that $\sum_{N=1}^{N_{\max }} N q_{I}(N)=\sum_{N=1}^{N_{\max }} N q_{V}(N)=1$. We use a limit, $N_{\max }$, for the number of point defects in a single defect, as an infinite sized defect cannot be produced in a single subcascade ${ }^{41,86}$.

As a first order approximation we can write the relaxation volumes of interstitial/vacancy clusters using their leading terms, $\Omega_{I}(N) / \Omega_{0} \sim N+b_{0}^{(I)} \sqrt{N} \log N+b_{1}^{(I)} \sqrt{N}$ and $\Omega_{V}(N) / \Omega_{0} \sim b_{0}^{(V)} N^{2 / 3}$. Then we can write down the relaxation volume per Frenkel pair for the cascade as

$$
\begin{aligned}
& \begin{array}{l}
\Omega_{r e l} / \Omega_{0} / N_{F P} \\
\text { where }
\end{array} 1+b_{0}^{(I)} w_{I, 0}+b_{1}^{(I)} w_{I, 1}+b_{0}^{(V)} w_{V} \\
& w_{I, 0}= \frac{\sum_{N=1}^{N_{\max }} \ln (N) N^{1 / 2-s_{I}}}{\sum_{N=1}^{N_{\max }} N^{1-s_{I}}} \\
& w_{I, 1}=\frac{\sum_{N=1}^{N_{\max }} N^{1 / 2-s_{I}}}{\sum_{N=1}^{N_{\max }} N^{1-s_{I}}} \\
& w_{V}=\frac{\sum_{N=1}^{N_{\max }} N^{2 / 3-s_{V}}}{\sum_{N=1}^{N_{\max }} N^{1-s_{V}}} .
\end{aligned}
$$

The coefficients $w_{I, 0}, w_{I, 1}, w_{V}$, which determine the relative importance of interstitial and vacancy contributions 


\begin{tabular}{l|cc|cc} 
& \multicolumn{2}{|c|}{$\Omega_{r e l} / \Omega_{0} / N^{2 / 3}$} & \multicolumn{2}{|c}{$\begin{array}{c}E_{f} / N^{2 / 3} \\
\end{array}$} \\
& fit to simulation data surface stress model & fit to simulation data surface energy model \\
\hline DFT & -0.49 & -0.66 & 7.18 & 7.08 \\
MNB & -0.50 & -0.41 & 7.35 & 7.14 \\
DND & -0.31 & -0.20 & 5.25 & 5.31 \\
CEA4 & -0.12 & -0.16 & 5.56 & 5.33
\end{tabular}

TABLE IX. The leading order constant for the relaxation volumes and formation energies (eV) in the limit of a large spherical void found by fitting to simulation data, as given in table VI and using a simple model using surface properties only (equations 30 and 32 ).

to the relaxation volume, are plotted in figure 11 . For the tungsten cascades we consider $N_{\max } \sim 1000, s_{I}=1.6$ and $s_{V}=2.0$ and so $w_{I, 0} \simeq 0.40, w_{I, 1} \simeq 0.15$ and $w_{V} \simeq$ 0.44 . We find in this work $b_{0}^{(I)}=0.008, b_{1}^{(I)}=0.738$ and $b_{0}^{(V)}=-0.50$ for the MNB potential ( see tables $\mathrm{V}$ and VI ), and so expect $\Omega_{\text {rel }} / \Omega_{0} / N_{F P} \sim 0.89$.

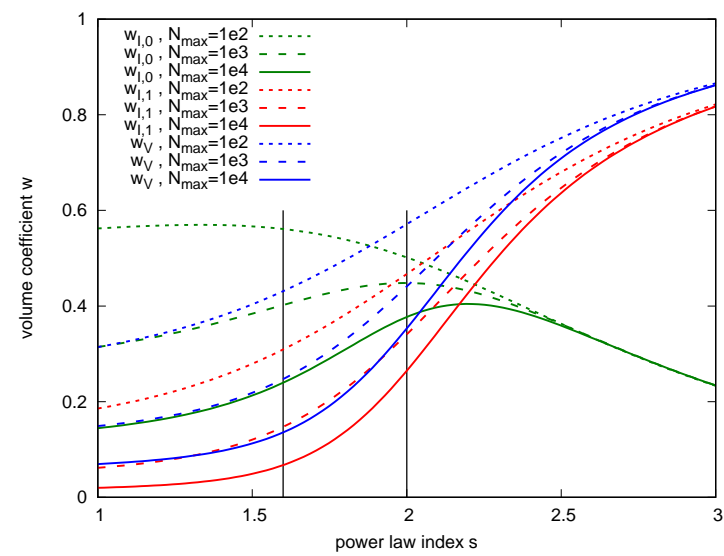

FIG. 11. Relaxation volume coefficients for cascades characterized by power-laws for the size-frequency distributions of interstitial loops and vacancy clusters, with power law indices $s_{I}$ and $s_{V}$. The relaxation volumes per point defect for a cascade can be written as $\Omega_{r e l} / \Omega_{0} / N_{F P}=1+b_{0}^{(I)} w_{I, 0}+$ $b_{1}^{(I)} w_{I, 1}+b_{0}^{(V)} w_{V}$ Vertical lines indicate the power indices measured using MD for $150 \mathrm{keV}$ PKA cascades in tungsten ${ }^{85}$.

We could get a slightly better estimate for the total cascade relaxation volume by using the $\Omega_{I / V}(N)$ given by the values for the smallest clusters in table III and the full expressions given by the fits in tables $\mathrm{VI}$, and $\mathrm{V}$ for larger clusters. This gives the values $\Omega_{r e l} / \Omega_{0} / N_{F P}=0.87$ for MNB. For DND and CEA4 the prefactor is 0.80 and 1.31 respectively. Though not a perfect match to the observed total relaxation volumes computed in section IID, this simple calculation returns that MNB has a larger relaxation volume than DND, and that both have a scaling factor a little under unity.

\section{IMPLICATIONS FOR MODELLING MICROSTRUCTURAL EVOLUTION UNDER IRRADIATION}

We noted earlier that, in agreement with the analysis performed in Refs. ${ }^{26,27}$, the fact that the relaxation volume of a self-interstitial atom defect is positive and fairly large whereas the relaxation volume of a vacancy is negative and relatively small, represents the fundamental reason why metals expand and swell under irradiation. The dynamics of accumulation of defects, involving their recombination and coalescence, gives rise to a fairly complex picture of microstructural evolution, where internal stresses and strains as well as the volume of a reactor component exposed to irradiation vary as functions of time. For example, some volumetric expansion occurs effectively instantaneously as a result of the generation of defects in collision cascades ${ }^{87-90}$, see Fig. 1, due to the fact that the total (positive) relaxation volume of all the self-interstitial defects produced in a cascade is greater than the (negative) relaxation volume of all the vacancies. This is confirmed by the analysis given in section III D above, which also shows that no notion of diffusionmediated microstructural evolution is required to arrive at the conclusion that the production of defects in cascades gives rise to swelling.

Using the equations for relaxation volumes of defect clusters given above, it is possible to evaluate the degree of volumetric expansion of a material even in the limit where the voids formed under irradiation are too small to be visible in an electron microscope. Also, the formulae for defect relaxation volumes are directly applicable for computing the volumetric expansion of an evolving microstructure simulated using object kinetic Monte Carlo ${ }^{9,91,92}$.

In the mesoscopic limit, the relaxation volumes of dislocation loops, of either self-interstitial and vacancy nature, can be evaluated as line integrals over the loop perimeters $^{20,93}$

$$
\begin{aligned}
& \Omega_{r e l}=-\frac{1}{2} \oint_{\Gamma} \mathbf{b} \cdot(\mathbf{r} \times d \mathbf{l}), \\
& \frac{d \Omega_{r e l}}{d t}=-\oint_{\Gamma} \mathbf{b} \cdot(\mathbf{v} \times d \mathbf{l}),
\end{aligned}
$$

where $\mathbf{r}$ and $\mathbf{v}$ are the position and velocity of a point on a dislocation line, and the choice of the sign before the integral depends on the Burgers vector convention ${ }^{20}$. 
Equation (37), together with the formula for the relaxation volume of a void or a gas bubble (28), enables evaluating volumetric swelling in the limit where radiation defect objects are mesoscopic or even macroscopic. In this limit, the degree of volumetric swelling may be assessed by analysing the relaxation volume of dislocation loops, which are often readily seen in electron microscope images, rather than the void component of microstructure.

The treatment of dynamics of diffusion-mediated evolution of microstructure is beyond the scope of this paper, although it would be appropriate to note that equation (37) for the rate of variation of relaxation volume of dislocations provides a link between diffusion-mediated models for microstructural evolution ${ }^{81,94-96}$, and the stress and strain based treatment of radiation induced volumetric swelling of a material given above in this paper.

\section{CONCLUSIONS}

In this paper we have computed the relaxation volumes of a broad variety of lattice defects in tungsten, using three empirical potentials and density functional theory. We have presented the data in a number of scatter plots, but have also presented tabulated data for empirical fits to the results. It is hoped that the data in this form is readily applicable to a range of coarse grained models, which involve either the elastic interactions between defects or the stresses and strains induced by the defect population.

We have found that there is some considerable variation in the absolute values of relaxation volumes of defects compared across the EAM potentials we have considered. This is because the elastic properties of defects were never considered in the parameter fitting. We were, however, able to identify some cross-potential trends. The relaxation volume per point defect varies according the specific configuration of the defect cluster, but for small defect clusters $(N<10)$ is likely to remain in the range $\pm 10 \%$. This means that knowing the point defect content of a cluster and its character ( vacancy- or interstitial- type ) is sufficient to predict the relaxation volume of a cluster. Larger defect clusters $(N>10)$ are most stable as dislocation loops, categorised by a Burgers vector, or in the case of vacancies are more stable as voids $\left(N<6 \times 10^{5}\right.$ for MNB or $N<3 \times 10^{6}$ for CEA4 $4^{72}$, and we find $N<8 \times 10^{6}$ for DND). $1 / 2\langle 111\rangle$ and $\langle 100\rangle$ dislocation loops and voids are sufficiently dissimilar to require their own representation of relaxation volume as a function of defect size, to predict their elastic properties.

We showed in section IID that the relaxation volume of a cascade is proportional to the number of Frenkel pairs it contains, with a positive coefficient around unity. The structure in the relaxation volume per point defect is to some extent averaged out by the range of sizes of defects produced in a cascade. The expected number of Frenkel pairs per cascade is itself proportional to the
PKA energy, according to the NRT formula ${ }^{97}$, a result broadly confirmed by MD simulation ${ }^{4,98}$, though the constant of proportionality is now widely held to be somewhat smaller than that given by the NRT model. The recent arc-dpa model ${ }^{99,100}$ predicts the number of Frenkel pairs per cascades produced in a defect-free material at low energy to be slightly above the line of proportionality seen at high energy, but we can still say that as a ruleof-thumb, the relaxation volume per cascade increases roughly linearly with the PKA energy. As the defect microstructure evolves, annihilation between vacancy- type and interstitial- type will reduce the total relaxation volume. This is a much more significant effect than deviations in proportionality of the total volume of initial defects to the PKA energy, but it will nevertheless preserve approximately the linear dependence of relaxation volume with Frenkel pair count. Coalescence of small defect clusters will have a smaller effect on the linear dependence of the relaxation volume with Frenkel pair count. As we have found a potential-dependent non-monotonic variation of the relaxation volume of individual dislocation loops with point defect count $N$, it is not clear at this point whether coalescence will increase or decrease the relaxation volume. DFT calculations of the dipole tensors of large loops may be able to answer this question in the future.

\section{ACKNOWLEDGMENTS}

This work has been carried out within the framework of the EUROfusion Consortium and has been received funding from Euratom research and training programme 2019-2020 under grant agreement No. 633053 and from the RCUK Energy Programme [grant number EP/P012450/1]. The views and opinions expressed herein do not necessarily reflect those of the European Commission. DNM acknowledges the support from high-performance computing facility MARCONI (Italy) through the EUROfusion HPC project AMPSTANI (2017-2019). AES acknowledges support from the Academy of Finland through project No. 311472.

\footnotetext{
${ }^{1}$ J. Marian, C.S. Becquart, C. Domain, S.L. Dudarev, M.R. Gilbert, R.J. Kurtz, D.R. Mason, K. Nordlund, A.E. Sand, L.L. Snead, T. Suzudo, and Wirth B.D. Recent advances in computational materials modeling of tungsten as plasma-facing material for fusion energy applications. Nuclear Fusion, 2016.

${ }^{2}$ D. Nguyen-Manh, M. Y. Lavrentiev, M. Muzyk, and S.L. Dudarev. First-principles models for phase stability and radiation defects in structural materials for future fusion power-plant applications. J Mater Sci, 47:7385 - 7398, 2012.

${ }^{3}$ S.L. Dudarev. Density functional theory models for radiation damage. Annual Review of Materials Research, 43:35-61, 2013.

${ }^{4}$ A.F. Calder and D.J. Bacon. A molecular dynamics study of displacement cascades in a-iron. Journal of Nuclear Materials, 207:25 - 45, 1993.

${ }^{5}$ R.E. Stoller and L.R. Greenwood. Subcascade formation in displacement cascade simulations: Implications for fusion reactor materials. J. Nucl. Mater, 271-272:57-62, 1999.

${ }^{6}$ A. E. Sand, S. L. Dudarev, and K. Nordlund. High-energy collision cascades in tungsten: Dislocation loops structure and clus-
} 
tering scaling laws. EPL (Europhysics Letters), 103(4):46003, 2013.

${ }^{7}$ L. K. Béland, P. Brommer, F. El-Mellouhi, J.-F. Joly, and N. Mousseau. Kinetic activation-relaxation technique. Phys. Rev. E, 84:046704, Oct 2011.

${ }^{8} \mathrm{H}$. Xu, Y. N. Osetsky, and R. E. Stoller. Self-evolving atomistic kinetic monte carlo: fundamentals and applications. Journal of Physics: Condensed Matter, 24(37):375402, 2012.

${ }^{9}$ D R Mason, X Yi, M A Kirk, and S L Dudarev. Elastic trapping of dislocation loops in cascades in ion-irradiated tungsten foils. Journal of Physics: Condensed Matter, 26(37):375701, 2014.

${ }^{10} \mathrm{~J}$. Marian and V. V. Bulatov. Stochastic cluster dynamics method for simulations of multispecies irradiation damage accumulation. Journal of Nuclear Materials, 415(1):84 - 95, 2011.

${ }^{11}$ A. Y. Dunn, L. Capolungo, E. Martinez, and M. Cherkaoui. Spatially resolved stochastic cluster dynamics for radiation damage evolution in nanostructured metals. Journal of Nuclear Materials, 443(1):128 - 139, 2013.

${ }^{12}$ X. Yi, A. E. Sand, D. R. Mason, M. A. Kirk, S. G. Roberts, K. Nordlund, and S. L. Dudarev. Direct observation of size scaling and elastic interaction between nano-scale defects in collision cascades. EPL (Europhysics Letters), 110(3):36001, 2015.

${ }^{13}$ S.L. Dudarev, D.R. Mason, E. Tarleton, P.-W. Ma, and A.E. Sand. Multi-scale model for stresses, strains and swelling of reactor components under irradiation. Nuclear Fusion, 58:126002, 2018.

${ }^{14}$ F. Hofmann, X. Song, T.-S. Jun, B. Abbey, M. Peel, J. Daniels, V. Honkimäki, and A.M. Korsunsky. High energy transmission micro-beam Laue synchrotron X-ray diffraction. Materials Letters, 64(11):1302 - 1305, 2010.

${ }^{15}$ F. Hofmann, D. Nguyen-Manh, M.R. Gilbert, C.E. Beck, J.K. Eliason, A.A. Maznev, W. Liu, D.E.J. Armstrong, K.A. Nelson, and S.L. Dudarev. Lattice swelling and modulus change in a helium-implanted tungsten alloy: X-ray micro-diffraction, surface acoustic wave measurements, and multiscale modelling. Acta Materialia, 89:352 - 363, 2015.

${ }^{16}$ W. Hertz, W. Waidelich, and H. Peisl. Lattice contraction due to quenching in vacancies in platinum and gold. Physics Letters, $43 \mathrm{~A}(3): 289-290,1973$.

${ }^{17}$ S.J Zinkle and N.M Ghoniem. Operating temperature windows for fusion reactor structural materials. Fusion Engineering and Design, 51-52:55 - 71, 2000.

${ }^{18} \mathrm{H}$ Bolt, V Barabash, G Federici, J Linke, A Loarte, J Roth, and K Sato. Plasma facing and high heat flux materials - needs for ITER and beyond. Journal of Nuclear Materials, 307-311, Part $1: 43-52,2002$.

${ }^{19}$ S.J. Zinkle and G.S. Was. Materials challenges in nuclear energy. Acta Materialia, 61(3):735 - 758, 2013. The Diamond Jubilee Issue.

${ }^{20}$ S.L. Dudarev and P.-W. Ma. Elastic fields, dipole tensors, and interaction between self-interstitial atom defects in bcc transition metals. Phys. Rev. Mater., 2:033602, 2018.

${ }^{21}$ P.-W Ma and S.L. Dudarev. Universality of point defect structure in body-centred cubic metals. Physical Review Materials, 3:013605, 2019.

${ }^{22}$ P.-W. Ma and S. L. Dudarev. Symmetry-broken self-interstitial defects in chromium, molybdenum, and tungsten. Physical Review Materials, 3:043606, 2019.

${ }^{23} \mathrm{~W}$. Schilling. Self-interstitial atoms in metals. Journal of $\mathrm{Nu}$ clear Materials, 69-70:465 - 489, 1978.

${ }^{24} \mathrm{~W}$. G. Wolfer. A novel approach to compute the relaxation volume of self-interstitials in metals. Journal of Physics: Metal Physics, 12:425 - 433, 1982.

${ }^{25}$ Y. Kraftmakher. Equilibrium vacancies and thermophysical properties of metals. Physics Reports, 299:79 - 188, 1998.

${ }^{26} \mathrm{~W}$. G. Wolfer. The dislocation bias. Journal of Computer-Aided Materials Design, 14:403 - 417, 2007.

${ }^{27}$ M. P. Surh, J. B. Sturgeon, and W. G. Wolfer. Void nucleation, growth, and coalescence in irradiated metals. Journal of Nuclear Materials, 378:86 - 97, 2008.
${ }^{28}$ R. Benedek, L. H. Yang, C. Woodward, and B. I. Min. Formation energy and lattice relaxation for point defects in $\mathrm{Li}$ and $\mathrm{Al}$. Phys. Rev. B, 45:2607-2612, Feb 1992.

${ }^{29} \mathrm{R}$ Pawellek, M Fahnle, C Elsasser, K M Ho, and C T Chan. First-principles calculation of the relaxation around a vacancy and the vacancy formation energy in $\mathrm{BCC} \mathrm{Li}$. Journal of Physics: Condensed Matter, 3(14):2451-2455, apr 1991.

${ }^{30}$ W. Frank, U. Breier, C. Elsässer, and M. Fähnle. Properties of monovacancies and self-interstitials in bcc Li: An ab initi pseudopotential study. Phys. Rev. B, 48:7676-7678, Sep 1993.

${ }^{31}$ W. Frank, U. Breier, C. Elsässer, and M. Fähnle. Firstprinciples calculations of absolute concentrations and selfdiffusion constants of vacancies in lithium. Phys. Rev. Lett., 77:518-521, Jul 1996.

${ }^{32}$ U. Breier, W. Frank, C. Elsässer, M. Fähnle, and A. Seeger. Properties of monovacancies and self-interstitials in bcc Na: An ab initio pseudopotential study. Phys. Rev. B, 50:5928-5936, Sep 1994.

${ }^{33}$ V. Schott, M. Fähnle, and P. A. Madden. Theory of selfdiffusion in alkali metals: I. results for monovacancies in Li, Na, and K. Journal of Physics: Condensed Matter, 12(7):11711194, feb 2000.

${ }^{34}$ M. Rieth, J.L. Boutard, S.L. Dudarev, T. Ahlgren, S. Antusch, N. Baluc, M.-F. Barthe, C.S. Becquart, L. Ciupinski, J.B. Correia, C. Domain, J. Fikar, E. Fortuna, C.-C. Fu, E. Gaganidze, T.L. Galán, C. García-Rosales, B. Gludovatz, H. Greuner, K. Heinola, N. Holstein, N. Juslin, F. Koch, W. Krauss, K.J. Kurzydlowski, J. Linke, Ch. Linsmeier, N. Luzginova, H. Maier, M.S. Martínez, J.M. Missiaen, M. Muhammed, A. Mu noz, M. Muzyk, K. Nordlund, D. Nguyen-Manh, P. Norajitra, J. Opschoor, G. Pintsuk, R. Pippan, G. Ritz, L. Romaner, D. Rupp, R. Schäublin, J. Schlosser, I. Uytdenhouwen, J.G. van der Laan, L. Veleva, L. Ventelon, S. Wahlberg, F. Willaime, S. Wurster, and M.A. Yar. Review on the EFDA programme on tungsten materials technology and science. Journal of Nuclear Materials, 417(1-3):463 - 467, 2011. Proceedings of ICFRM-14.

${ }^{35}$ M. Rieth, S.L. Dudarev, and S.M. Gonzalez de Vicente et al. Recent progress in research on tungsten materials for nuclear fusion applications in Europe. Journal of Nuclear Materials, 432(1-3):482 - 500, 2013.

${ }^{36}$ F. H. Featherston and J. R. Neighbours. Elastic constants of tantalum, tungsten, and molybdenum. Phys. Rev., 130:13241333, May 1963.

${ }^{37}$ A. Stukowski. Visualization and analysis of atomistic simulation data with OVITO - the open visualization tool. Model. Simul. Mater. Sci. Eng, 18:015012, 2010.

${ }^{38}$ R. A. Johnson. Point-defect calculations for tungsten. Phys. Rev. B, 27:2014-2018, Feb 1983.

${ }^{39}$ D. Kato, H. Iwakiri, and K. Morishita. Formation of vacancy clusters in tungsten crystals under hydrogen-rich condition. Journal of Nuclear Materials, 417(1):1115 - 1118, 2011. Proceedings of ICFRM-14.

${ }^{40}$ P. M. Derlet, D. Nguyen-Manh, and S. L. Dudarev. Multiscale modeling of crowdion and vacancy defects in body-centeredcubic transition metals. Phys. Rev. B, 76:054107, Aug 2007.

${ }^{41}$ A. E. Sand, D. R. Mason, A. De Backer, X. Yi, S. L. Dudarev, and K. Nordlund. Cascade fragmentation: deviation from power law in primary radiation damage. Materials Research Letters, 5(5):357-363, 2017.

${ }^{42}$ D.R. Mason, A.E. Sand, X. Yi, and S.L. Dudarev. Direct observation of the spatial distribution of primary cascade damage in tungsten. Acta Materialia, 144:905 - 917, 2018.

${ }^{43}$ D. R. Mason, X. Yi, A. E. Sand, and S. L. Dudarev. Experimental observation of the number of visible defects produced in individual primary damage cascades in irradiated tungsten. EPL (Europhysics Letters), 122(6):66001, 2018.

${ }^{44}$ M.-C. Marinica, L. Ventelon, M. R. Gilbert, L. Proville, S. L. Dudarev, J. Marian, G. Bencteux, and F. Willaime. Interatomic potentials for modelling radiation defects and dislocations in tungsten. Journal of Physics: Condensed Matter, 
25(39):395502, 2013.

${ }^{45}$ G. J. Ackland, M. I. Mendelev, D. J. Srolovitz, S. Han, and A.V. Barashev. Development of an interatomic potential for phosphorus impurities in $\alpha$-iron. Journal of Physics: Condensed Matter, 16(27):S2629, 2004.

${ }^{46}$ D.R. Mason, D. Nguyen-Manh, and C.S. Becquart. An empirical potential for simulating vacancy clusters in tungsten. Journal of Physics: Condensed Matter, 29(50):505501, 2017.

${ }^{47}$ M. W. Finnis and J. E. Sinclair. A simple empirical n-body potential for transition metals. Philosophical Magazine A, 50(1):45-55, 1984.

${ }^{48}$ G.J. Ackland and R. Thetford. An improved n-body semiempirical model for body-centred cubic transition metals. Philosophical Magazine A, 56:15-30, 1987.

${ }^{49}$ A. M. Kossevich. The Crystal Lattice. Wiley-VCH, Berlin, 1999.

${ }^{50}$ C.B. Barber, D.P. Dobkin, and H.T. Huhdanpaa. The quickhull algorithm for convex hulls. ACM Trans. on Mathematical Software, 22(4):469-483, 1996.

${ }^{51}$ Note that this same excess linear correction $r$ would be required to find the volume of a periodic supercell from the convex hull of the atoms contained within.

${ }^{52} \mathrm{H}$. Kanzaki. Point defects in face-centred cubic lattice-I distortion around defects. J. Phys. Chem. Solids, 2:24-36, 1957.

${ }^{53}$ C. Domain and C.S. Becquart. Ab initio calculations of defects in Fe and dilute Fe-Cu alloys. Phys. Rev. B, 65:024103, 2001.

${ }^{54}$ D.M. Barnett. The precise evaluation of derivatives of the anisotropic elastic Green's functions. Phys. Stat. Solidi, 49:741$748,1972$.

${ }^{55} \mathrm{C}$. Varvenne and E. Clouet. Elastic dipoles of point defects from atomistic simulations. Phys. Rev. B, 96:224103, 2017.

${ }^{56}$ G. Leibfried and N. Breuer. Point Defects in Metals. Springer, Berlin, 1978.

${ }^{57}$ C. Varvenne, F. Bruneval, M.-C. Marinica, and E. Clouet. Point defect modeling in materials: Coupling $a b$ initio and elasticity approaches. Phys. Rev. B, 88:134102, 2013.

${ }^{58}$ F.H. Featherstone and J.R. Neighbours. Elastic constants of tantalum, tungsten, and molybdenum. Phys. Rev., 130:13241333, 1963.

${ }^{59}$ F.R. de Boer, R. Boom, W.C.M. Mattens, A.R. Miedema, and A.K. Niessen. Cohesion in Metals. North-Holland, Amsterdam, 1988.

${ }^{60} \mathrm{G}$. Kresse and J. Hafner. Ab initio molecular dynamics for liquid metals. Phys. Rev. B, 47:558-561, Jan 1993.

${ }^{61}$ G. Kresse and J. Furthmüller. Efficient iterative schemes for ab initio total-energy calculations using a plane-wave basis set. Phys. Rev. B, 54:11169-11186, Oct 1996.

${ }^{62} \mathrm{G}$. Kresse and J. Furthmüller. Efficiency of ab-initio total energy calculations for metals and semiconductors using a plane-wave basis set. Computational Materials Science, 6(1):15 - 50, 1996.

${ }^{63}$ D. Nguyen-Manh, A. P. Horsfield, and S.L. Dudarev. Selfinterstitial atom defects in bcc transition metals: Group-specific trends. Physical Review B, 73:020101, 2006.

${ }^{64}$ D. Nguyen-Manh and S.L. Dudarev. Trapping of He clusters by inert-gas impurities in tungsten: First-principles predictions and experimental validation. Nuclear Instruments and Methods in Physics Research Section B: Beam Interactions with Materials and Atoms, 352:86 - 91, 2015. Proceedings of the 12th International Conference on Computer Simulation of Radiation Effects in Solids, Alacant, Spain, 8-13 June, 2014.

${ }^{65}$ A.E. Sand, J. Dequeker, C.S. Becquart, C. Domain, and K. Nordlund. Non-equilibrium properties of interatomic potentials in cascade simulations in tungsten. Journal of Nuclear Materials, 470(Supplement C):119 - 127, 2016.

${ }^{66}$ J. P. Perdew, K. Burke, and M. Ernzerhof. Generalized gradient approximation made simple. Phys. Rev. Lett., 77:3865-3868, Oct 1996.

${ }^{67}$ R. Alexander, M.-C. Marinica, L. Proville, F. Willaime, K. Arakawa, M. R. Gilbert, and S. L. Dudarev. Ab initio scaling laws for the formation energy of nanosized interstitial defect clusters in iron, tungsten, and vanadium. Phys. Rev. B,
94:024103, Jul 2016.

${ }^{68}$ M.-C. Marinica, F. Willaime, and J.-P. Crocombette. Irradiation-induced formation of nanocrystallites with $\mathrm{C} 15$ Laves phase structure in bcc iron. Phys. Rev. Lett, 108:025501, 2012.

${ }^{69}$ N. Gao, W. Setyawan, R.J. Kurtz, and Z. Wang. Effects of applied strain on nanoscale self-interstitial cluster formation in bcc iron. J. Nucl. Mater., 493:62-68, 2017.

${ }^{70}$ X. Yi, M.L. Jenkins, M. Brice no, S.G. Roberts, Z. Zhou, and M.A. Kirk. In situ study of self-ion irradiation damage in W and W-5Re at 500C. Philosophical Magazine, 93(14):1715-1738, 2013.

${ }^{71}$ J. Fikar and R. Gröger. Shape of small prismatic dislocation loops in tungsten and iron. In Materials Structure 85 Micromechanics of Fracture VIII, volume 258 of Solid State Phenomena, pages 97-101. Trans Tech Publications, 12017.

72 J. Fikar, R. Schäublin, D. R. Mason, and D. Nguyen-Manh. Nano-sized prismatic vacancy dislocation loops and vacancy clusters in tungsten. Nuclear Materials and Energy, 16:60 $65,2018$.

${ }^{73}$ M R Gilbert, S L Dudarev, P M Derlet, and D G Pettifor. Structure and metastability of mesoscopic vacancy and interstitial loop defects in iron and tungsten. Journal of Physics: Condensed Matter, 20(34):345214, 2008.

${ }^{74} \mathrm{P}$. Diaconis and B. Efron. Computer-intensive methods in statistics. Scientific American, 248:116-130, 1983.

${ }^{75}$ The Quest for Clean Energy: IAEA Challenge on Materials for Fusion, https://challenge.iaea.org/challenges/2018-NAMat-Fusion/about, April 2018.

${ }^{76}$ C. Björkas, K. Nordlund, and S. L. Dudarev. Modelling radiation effects using the ab-initio based tungsten and vanadium potentials. Nucl. Instr. Meth. B, 267:3204-3208, 2009.

${ }^{77}$ A.E. Sand and K. Nordlund. On the lower energy limit of electronic stopping in simulated collision cascades in Ni,Pd and Pt. Journal of Nuclear Materials, 456:99-105, 2015.

${ }^{78}$ H.J.C. Berendsen, J.P.M. Postma, W.F. van Gunsteren, A. DiNola, and J.R. Haak. Molecular dynamics with coupling to an external bath. J. Chem. Phys., 81:3684, 1998.

${ }^{79}$ A. Stukowski, V.V. Bulatov, and A. Arsenlis. Automated identification and indexing of dislocations in crystal interfaces. Model. Simul. Mater. Sci. Eng., 20:085007, 2012.

${ }^{80}$ S.L. Dudarev and A.P. Sutton. Elastic interactions between nano-scale defects in irradiated materials. Acta Materialia, 125:425 - 430, 2017.

${ }^{81}$ W. G. Wolfer and M. Ashkin. Stress-induced diffusion of point defects to spherical sinks. Journal of Applied Physics, 46(2):547 $-557,1975$.

${ }^{82} \mathrm{~T}$. Frolov and Y. Mishin. Temperature dependence of the surface free energy and surface stress: An atomistic calculation for cu(110). Phys. Rev. B, 79:045430, 2009.

${ }^{83} \mathrm{G}$. Bonny, P. Grigorev, and D. Terentyev. On the binding of nanometric hydrogen-helium clusters in tungsten. Journal of Physics: Condensed Matter, 26(48):485001, 2014.

${ }^{84}$ J.F. Ziegler, J.P. Biersack, and U. Littmark. The stopping and range of ions in solids. Pergamon, 1982.

${ }^{85}$ A. E. Sand, M. J. Aliaga, M. J. Caturla, and K. Nordlund. Surface effects and statistical laws of defects in primary radiation damage: Tungsten vs. iron. EPL (Europhysics Letters), 115(3):36001, 2016.

${ }^{86}$ A. De Backer, A. E. Sand, K. Nordlund, L. Luneville, D. Simeone, and Dudarev S. L. Subcascade formation and defect cluster size scaling in high-energy collision events in metals. EPL (Europhysics Letters), 115(2):26001, 2016.

${ }^{87} \mathrm{C}$. H. Woo and B. N. Singh. The concept of production bias and its possible role in defect accumulation under cascade damage conditions. physica status solidi (b), 159:609 - 616, 1990.

${ }^{88} \mathrm{C}$. H. Woo and B. N. Singh. Production bias due to clustering of point defects in irradiation-induced cascades. Philosophical Magazine A, 65:889 - 912, 1992.

${ }^{89}$ C. H. Woo, B. N. Singh, and F. A. Garner. Production bias: 
a proposed modification of the driving force for void swelling under cascade damage conditions. Journal of Nuclear Materials, 191-194:1224 - 1228, 1992.

${ }^{90}$ C. H. Woo, A. A. Semenov, and F. A. Garner. Analysis of microstructural evolution driven by production bias. Journal of Nuclear Materials, 206:170 - 199, 1993.

${ }^{91}$ C. Domain, C.S. Becquart, and L. Malerba. Simulation of radiation damage in fe alloys: an object kinetic Monte Carlo approach. Journal of Nuclear Materials, 335(1):121 - 145, 2004

${ }^{92}$ R.E. Stoller, S.I. Golubov, C. Domain, and C.S. Becquart. Mean field rate theory and object kinetic Monte Carlo: A comparison of kinetic models. Journal of Nuclear Materials, 382(2):77 - 90, 2008. Microstructural Processes in Irradiated Materials.

${ }^{93}$ I. Rovelli, S. L. Dudarev, and A. P. Sutton. Statistical model for diffusion-mediated recovery of dislocation and point-defect microstructure. Physical Review E, 98:043002, 2018.

${ }^{94}$ I. G. Margvelashvili and Z. K. Saralidze. Influence of an elastic field of a dislocation on steady-state diffusion fluxes of point defects. Soviet Physics: Solid State, 15:1774 - 1776, 1974.

${ }^{95} \mathrm{P}$. T. Heald. The preferential trapping of interstitials at dislocations. Philosophical Magazine, 31:551 - 558, 1975.

${ }^{96}$ A. D. Brailsford and R. Bullough. The theory of sink strengths. Philosophical Transactions of the Royal Society, 302:87 - 137, 1981.

${ }^{97}$ M.J. Norgett, M.T. Robinson, and I.M. Torrens. A proposed method of calculating displacement dose rates. Nuclear Engineering and Design, 33(1):50 - 54, 1975.

${ }^{98}$ C.S. Becquart, C. Domain, A. Legris, and J.C Van Duysen. Influence of the interatomic potentials on molecular dynamics simulations of displacement cascades. Journal of Nuclear Materials, 280(1):73 - 85, 2000.

${ }^{99}$ K. Nordlund, S. Zinkle, A.E. Sand, F. Granberg, R.S. Averback, R. Stoller, T. Suzudo, L. Malerba, F. Banhart, W. Weber, F. Willaime, S.L. Dudarev, and D. Simeone. Improving atomic displacement and replacement calculations with physically realistic damage models. Nat. Comm., 9:1048, 2018.

${ }^{100}$ K. Nordlund, S. Zinkle, A.E. Sand, F. Granberg, R.S. Averback, R. Stoller, T. Suzudo, L. Malerba, F. Banhart, W. Weber, F. Willaime, S.L. Dudarev, and D. Simeone. Primary radiation damage: A review of current understanding and models. Journal of Nuclear Materials, 512:450-479, 2018.

\section{Appendix A: Average surface energy}

In this appendix we find the average surface energy suitable for a spherical void given calculated values of the surface energy on facets. As we are working with cubic crystals, we expand the surface energy in cubic harmonics

$$
Y_{m n}(x, y, z)=\left(x^{4}+y^{4}+z^{4}\right)^{m}\left(x^{2} y^{2} z^{2}\right)^{n},
$$

where $x, y, z$ are direction cosines, so that the surface energy at a general direction is interpolated as

$$
g(x, y, z)=\sum_{m n} a_{m n} Y_{m n}(x, y, z)
$$

We can fit available surface data to the lowest orders of $Y_{m n}$. It is most common in the literature to see data for $\langle 110\rangle,\langle 100\rangle,\langle 211\rangle$, and $\langle 111\rangle$ planes, in which case it suffices to take $0 \leq m, n \leq 1$. Writing the surface energy for the $\langle 110\rangle$ plane as $\gamma_{\langle 110\rangle}$, and similarly for the others, we find

$$
\begin{aligned}
& a_{00}=-\gamma_{\langle 100\rangle}+2 \gamma_{\langle 110\rangle} \\
& a_{10}=2\left(\gamma_{\langle 100\rangle}-\gamma_{\langle 110\rangle}\right) \\
& a_{01}=27\left(\gamma_{\langle 100\rangle}+3 \gamma_{\langle 111\rangle}-4 \gamma_{\langle 211\rangle}\right) \\
& a_{11}=-54\left(\gamma_{\langle 100\rangle}+3 \gamma_{\langle 111\rangle}-6 \gamma_{\langle 211\rangle}+2 \gamma_{\langle 110\rangle}\right)
\end{aligned}
$$

The spherically-averaged surface energy, $\gamma$, can be found by integrating over the surface of the sphere:

$$
\begin{aligned}
\gamma & =\frac{1}{4 \pi} \int_{\theta=0}^{\pi} \int_{\phi=0}^{2 \pi} g(\sin \theta \cos \phi, \sin \theta \sin \phi, \cos \theta) \sin \theta d \theta d \phi \\
& =a_{00}+\frac{a_{01}}{105}+\frac{3 a_{10}}{5}+\frac{a_{11}}{231} \\
& =\frac{1}{385}\left(86 \gamma_{\langle 100\rangle}+128 \gamma_{\langle 110\rangle}+27 \gamma_{\langle 111\rangle}+144 \gamma_{\langle 211\rangle}\right) .
\end{aligned}
$$

It should be noted that two assumptions are made here, firstly that voids are unfacetted, and secondly that the surface energy varies smoothly with the orientation of the facet, so this interpolation should not be applied uncritically to other cases. However, this simple expression does give a single value for surface energy suitable for use in our analytical calculations of void relaxation volumes. 